\title{
Cryptocurrencies and Investment Diversification: Empirical Evidence from Seven Largest Cryptocurrencies
}

\author{
Nguyen Phuc Canh'1, Nguyen Quang Binh ${ }^{2}$, Su Dinh Thanh ${ }^{3}$ \\ ${ }^{1}$ School of Banking, University of Economics Ho Chi Minh City, Ho Chi Minh City, Vietnam \\ ${ }^{2}$ University of Economics Ho Chi Minh City, Ho Chi Minh City, Vietnam \\ ${ }^{3}$ School of Public Finance, University of Economics Ho Chi Minh City, Ho Chi Minh City, Vietnam \\ Email: canhnguyen@ueh.edu.vn,binhnq@ueh.edu.vn,dinhthanh@ueh.edu.vn
}

How to cite this paper: Canh, N.P., Binh, N.Q. and Thanh, S.D. (2019) Cryptocurrencies and Investment Diversification: Empirical Evidence from Seven Largest Cryptocurrencies. Theoretical Economics Letters, 9, 431-452. https://doi.org/10.4236/tel.2019.93031

Received: November 5, 2018

Accepted: March 3, 2019

Published: March 6, 2019

Copyright $\odot 2019$ by author(s) and Scientific Research Publishing Inc. This work is licensed under the Creative Commons Attribution International License (CC BY 4.0).

http://creativecommons.org/licenses/by/4.0/

\begin{abstract}
The study examines the diversification capability of seven cryptocurrencies with the largest market size against risks from economic factors as oil price, gold price, interest rate, USD strength, and S\&P500. Using the weekly data of Bitcoin, Litecoin, Ripple, Stellar, Monero, Dash, and Bytecoin in the period Aug/2014-Jun/2018, the study finds that there are structural breaks and ARCH disturbance in each cryptocurrency, suggesting a systematic risk within the cryptocurrency market. However, the causality between cryptocurrencies and economic factors is undirected. Interestingly, our findings show that cryptocurrencies are insignificant correlations with economic factors. The result implies that cryptocurrencies can not be assumed as financial assets to hedge systematic risks from economic factors.
\end{abstract}

\section{Keywords}

Cryptocurrency, Economic Factors, Systematic Risk

\section{Introduction}

The cryptocurrencies with a decentralized and open-source technology have extensively received attention from finance literature in recent years [1]. The fact is true that some financial institutions, public organizations and governments have recognized Bitcoin and other cryptocurrencies as official financial assets [2]. From the original objective as an alternative payment system independent of any central banks, the popularity of cryptocurrencies has tremendously received much attention from the literature due to their increased capitalization values. 
However, because of lacking foundational theories, linkages between cryptocurrencies and economic factors are still open to debate.

Some studies have focused on the volatility of cryptocurrency prices, especially the Bitcoin [3]-[10]. Other studies have emphasized the relationships between Bitcoin price and economic factors. $\mathrm{Su}, \mathrm{Li}$, Tao, and $\mathrm{Si}$ [11] showed that there have been four explosive bubbles in China and the U.S. market during the periods of the huge surges of Bitcoin prices and the shocks from foreign or domestic markets. Concerning Bitcoin and other assets [12] [13] [14] [15] found that the fundamental price of Bitcoin is close to zero. About the relation between cryptocurrencies, for instance, Bitcoin and Ethereum [16] [17] unveiled clear bubble behaviours during the time after 2013. Gandal, Hamrick, Moore, and Oberman [18] added that the suspicious trading resulted in an unprecedented spike of the USD-BTC exchange rate in late 2013.

Interestingly, as a hedge instrument against market-specific risk and uncertainty, Bitcoin may be a priority choice in portfolio management for financial markets [11] [19] [20] [21]. Some arguments show that the average monthly volatility of Bitcoin returns is higher than for gold or a set of foreign currencies indexed by dollars [22]. The Bitcoin price is more sensitive to changes in economic and market factors in the short-run, but less sensitive to technological factors in the long-run [23]. As in Al-Yahyaee, Mensi, and Yoon [24], the Bitcoin market is easy to be broken in comparison to other currencies markets, while Gajardo et al. [2] show that Bitcoin has a greater multifractal spectrum than other assets on its cross-correlation with the WTI, the Gold and the DJIA. Concerning the role of other cryptocurrencies, Ciaian, Rajcaniova, and Kancs [25] revealed that Bitcoin seems to be less affected macro-financial indicators in comparison to the altcoins price formation. On the contrary, Ciaian et al. [25] show that relationships among cryptocurrencies are complex, especially in the context of ICOs leading to a huge of cryptocurrencies available [26].

This study contributes to the literature by shedding the light on the capability of seven cryptocurrencies with the largest market capitalization in hedging against the systematic risks in line with economic factors. Specifically, the Granger causality tests between each cryptocurrency with economic factors show that the oil price, and the USD index cause most of the selected cryptocurrencies. While only BTC and LTC are among the cryptocurrencies, which cause the oil price, the USD index, the S\&P500 index and the gold price, respectively. In addition, there exist structural breaks and $\mathrm{ARCH}$ disturbance in the price of each cryptocurrency, suggesting a systematic risk within cryptocurrency markets. Moreover, the USD index has negative effects on all seven cryptocurrencies, while other economic factors have inconsistent effects on all cryptocurrencies. The results imply that the cryptocurrencies are likely impacted by economic factors other than a hedge for economic factors.

Next section presents the methodology and data. The results and discussions are in Section 3. Some conclusions are remarked in the final section. 


\section{Methodology and Data}

The study surveys all cryptocurrency markets and collects the daily closing price of each cryptocurrency and come up to 20 largest cryptocurrencies. Matching each cryptocurrency together with economic factors to find the longest time span possible, the study narrows down to seven cryptocurrencies in terms of largest market capitalization including Bitcoin, Litecoin, Ripple, Stellar, Monero, Dash, and Bytecoin in the period from 8 Aug 2014-7 June 2018. Economic indicators are proxied by WTI Oil price, Gold price, S\&P500 index, LIBOR, and USD index. The weekly data of WTI Oil price, S\&P500 index, Gold price, LIBOR (one month), and the bid price of USD index are collected from Thomson Reuter and Fred. All variables are taken by logarithm to reduce heteroskedasticity, except for LIBOR. Definitions, sources, and statistical descriptions of variables are presented in Table 1. The data of cryptocurrencies is collected from Coinmarketcap in Aug/2018. The LIBOR is collected from Federal Reserve Economic Data St. Louis Fed (FRED). All remained economic factors are collected from Thomson Reuter.

In this study, we collect the weekly data of all variables to enlarge the time span of the sample. In which, the weekly close values of all variables are used. Table 1 shows the primary data before taking logarithm. Bitcoin has highest average price then Dash, Monero, and Litecoin in the followings. To examine linkages between cryptocurrencies and world economic indicators, the study conducts Granger causality tests for each of pair variables. To detect the associations of cryptocurrencies with systematic risks, the study uses the $\operatorname{GARCH}(1,1)$ based on the existence of ARCH disturbance. $\operatorname{GARCH}(1,1)$ is formed as followings.

$$
Y_{t}=\beta_{0}+\beta_{i} X_{t}+\epsilon_{t}
$$

Table 1. Data description (primary data).

\begin{tabular}{cccccccc}
\hline Vars. & Definitions & Data & Obs. & Mean & Std. Dev. & Min & Max \\
\hline OIL & WTI Oil Price & Daily closed price & 204 & 53.713 & 13.440 & 29.42 & 97.65 \\
SP500 & SP500 Index & Daily closed index & 204 & 2250.556 & 262.696 & 1864.78 & 2872.87 \\
GOLD & Gold Price & Daily closed price & 204 & 1236.067 & 71.912 & 1058.41 & 1366.4 \\
LIBOR & LIBOR 1 Month & Daily closed interest & 204 & 0.723 & 0.582 & 0.152 & 2.098 \\
USD & USD Index Bid & Daily closed index & 204 & 94.820 & 4.310 & 81.424 & 103.01 \\
BTC & Bitcoin & Daily closed price & 204 & 2480.591 & 3732.954 & 208.1 & $17,706.9$ \\
XRP & Ripple & Daily closed price & 204 & 0.181 & 0.396 & 0.004 & 3.05 \\
LTC & Litecoin & Daily closed price & 204 & 34.947 & 61.386 & 1.4 & 299.78 \\
XLM & Stellar & Daily closed price & 204 & 0.056 & 0.129 & 0.0014 & 0.678 \\
XMR & Monero & Daily closed price & 204 & 51.295 & 93.456 & 0.256 & 394.78 \\
DAS & Dash & Daily closed price & 204 & 132.222 & 238.510 & 1.28 & 1179.01 \\
BCN & Bytecoin & Daily closed price & 204 & 0.001 & 0.002 & 0.000008 & 0.014 \\
\hline
\end{tabular}

Note: Time period: 8 Aug 2014-7 June 2018 due to the availability of Cryptocurrency prices from Coinmarketcap [from Aug 2014]. Source: Coinmarketcap, Fred, Thomson Reuters. 


$$
\begin{gathered}
\epsilon_{t} \mid \varphi_{t-1} \sim N\left(0, \partial_{t}^{2}\right) \\
\partial_{t}^{2}=\gamma+\alpha_{1} \epsilon_{t-1}^{2}+\delta_{1} \partial_{t-1}^{2}
\end{gathered}
$$

where: $Y$ is each cryptocurrency; $X$ is a set of economic factors including oil price, SP500, gold price, USD index, and LIBOR. $\beta$ is coefficient. $\epsilon$ is conditional error term. $\partial^{2}$ is GARCH term. $\epsilon^{2}$ is ARCH term. To check robustness, the study employs dynamic conditional correlation Multivariate GARCH model (Multivariate Autoregressive Conditionally Heteroskedastic-MGARCH). Due to the existence of ARCH disturbance and structural breaks in variables, MGARCH is more flexible than the conditional correlation MGARCH model, and more parsimonious than the diagonal vech MGARCH model [27] [28] [29]. The estimated results of conditional correlations from DCC MGARCH [30] [31] between each cryptocurrency and economic factors are helpful in detecting the associations between cryptocurrencies and economic factors.

The DCC GARCH model is given:

$$
\begin{gathered}
Y_{t}=\alpha X_{t}+\epsilon_{t} \\
\epsilon_{t}=H_{t}^{1 / 2} \gamma_{t}
\end{gathered}
$$

where: $Y$ is cryptocurrency; $X$ is a set of economic factors; $H$ is the Cholesky factor of the time-varying conditional covariance matrix; $\gamma_{t}$ is the vector of normal, independent, and identically distributed innovations.

\section{Results and Discussions}

\subsection{Basic Results}

Figure 1 shows that Bitcoin has the highest price with the peak at the end of 2017. All six other cryptocurrencies are the same patterns in this period. The US stock market (S\&P500) has a stable trend, while gold price and USD index show a small fluctuation during this time. Oil price steadily decreases from 2014 until 2015 before increasing until 2018. LIBOR shows a steadily increasing trend, especially from 2015 (Figures 1-4).

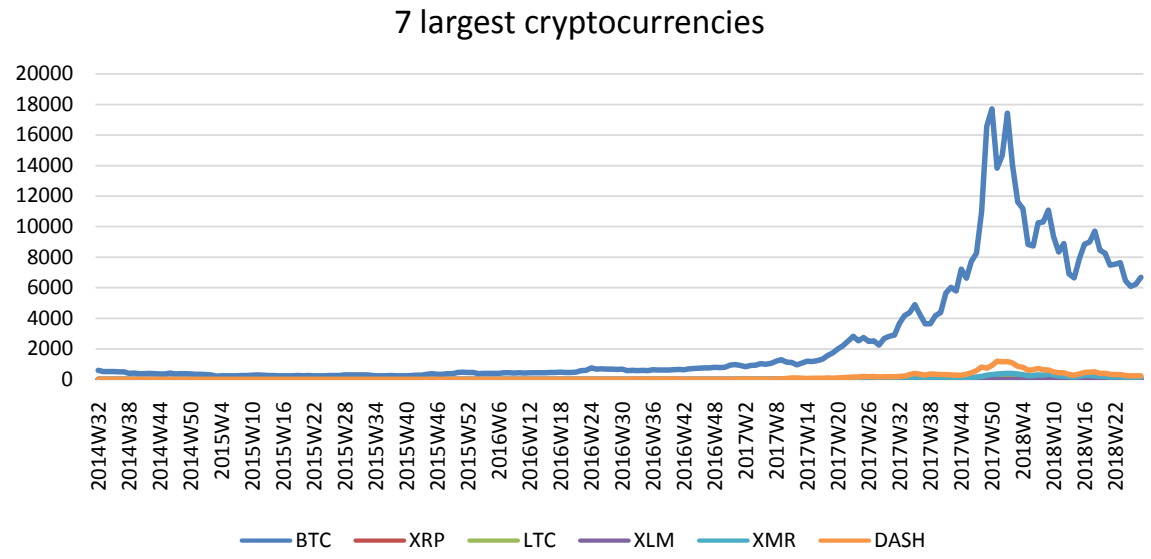

Figure 1. Price of 7 largest cryptocurrencies. 
Global Economic factors

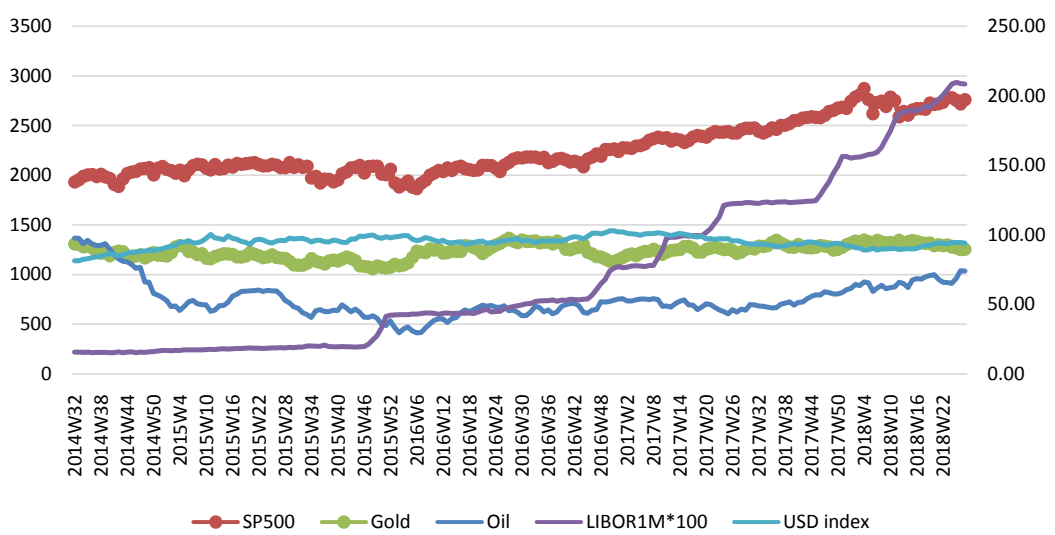

Figure 2. Economic factors in the period Aug/2014-Jun/2018 (SP500 and Gold are left axis; Old, LIBOR $(1 \mathrm{M}) \times 100$ and USD index are right axis).
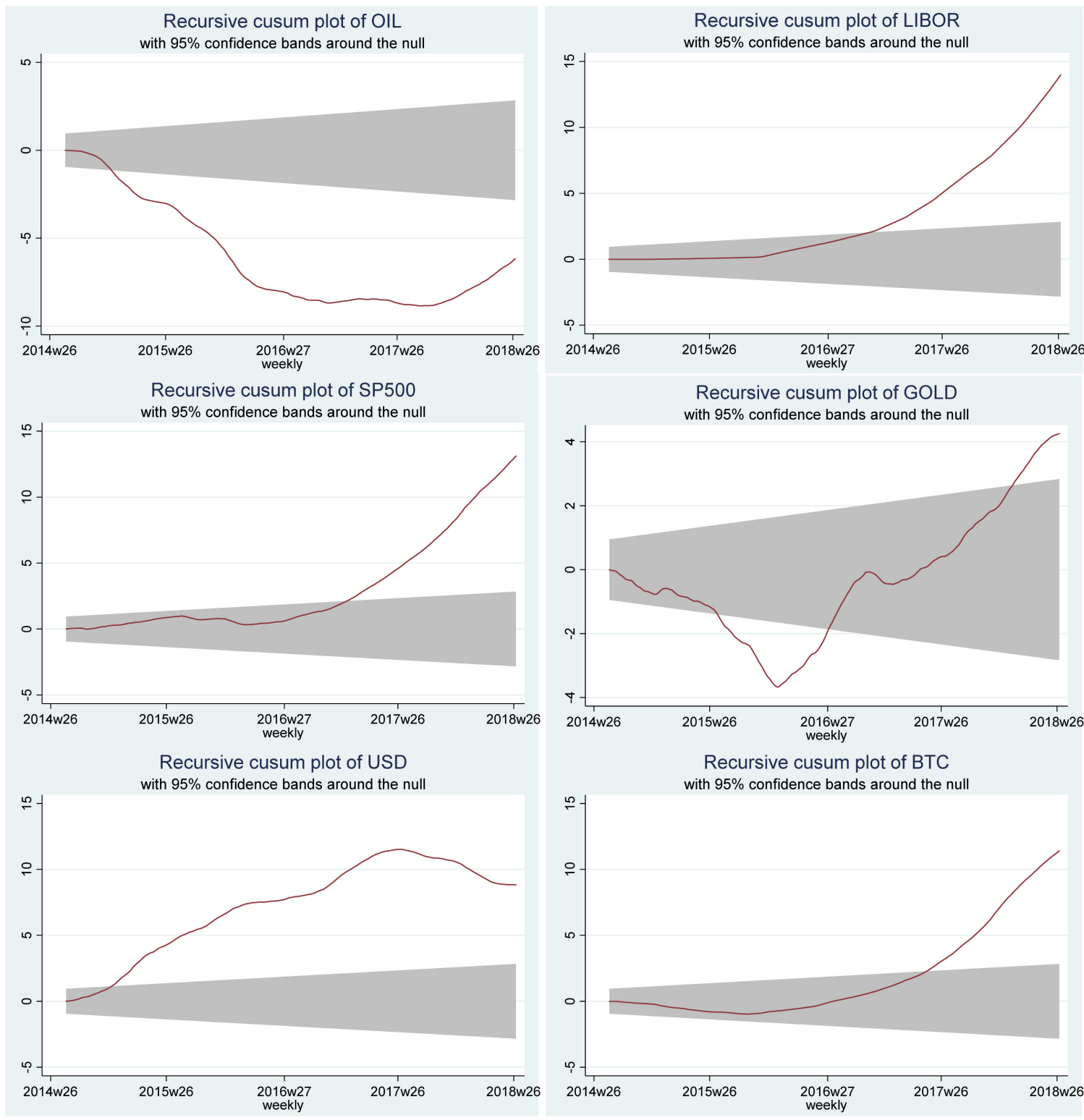

Recursive cusum plot of GOLD

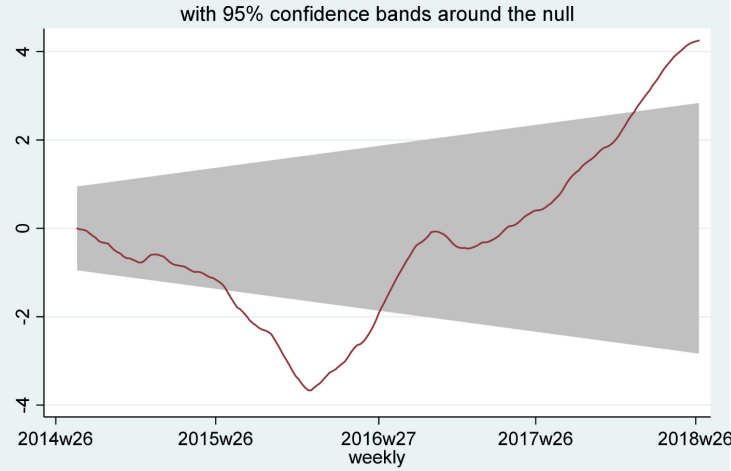

Recursive cusum plot of BTC with $95 \%$ confidence bands around the null

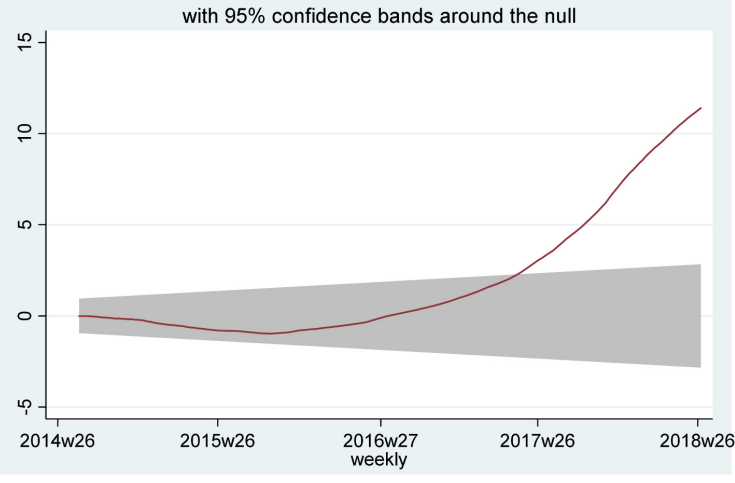


Recursive cusum plot of LTC

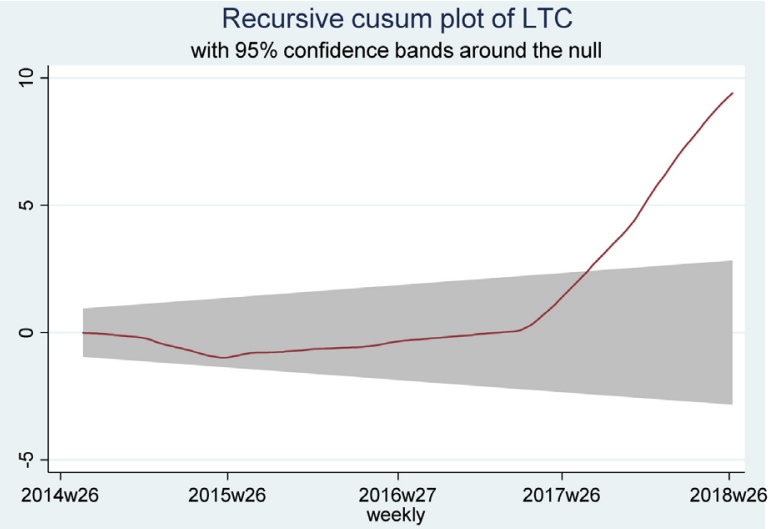

Recursive cusum plot of XLM

with $95 \%$ confidence bands around the null

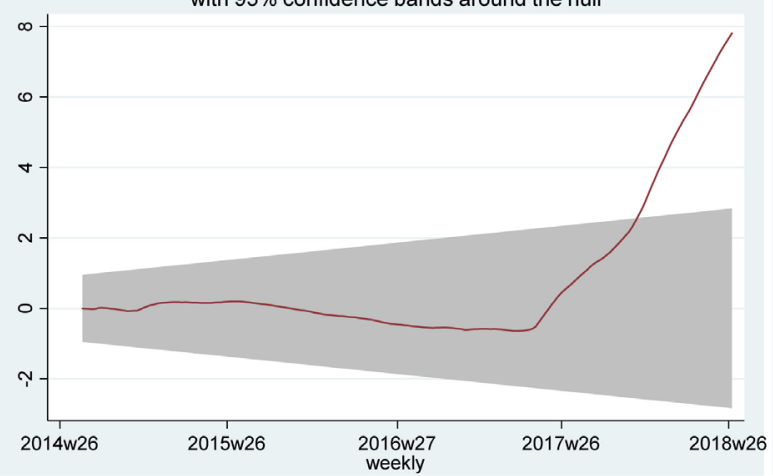

Recursive cusum plot of DASH

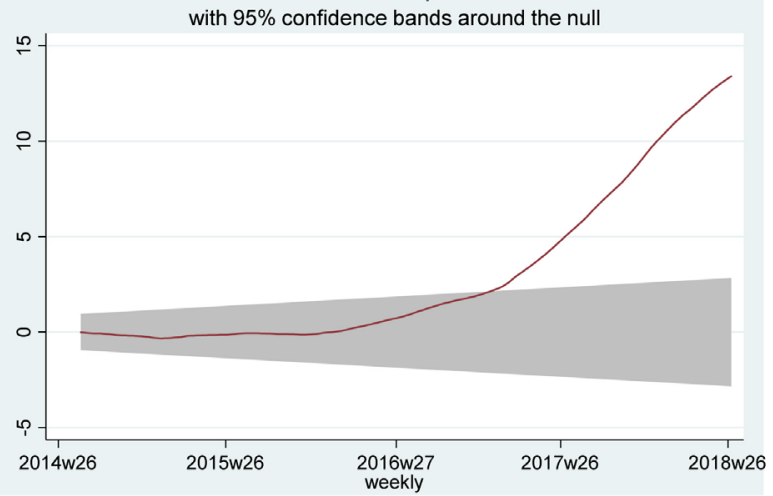

Recursive cusum plot of XRP

with $95 \%$ confidence bands around the null

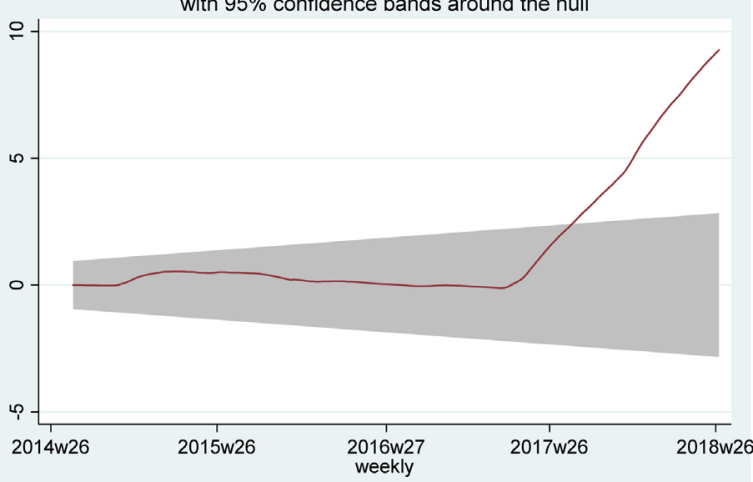

Recursive cusum plot of XMR

with $95 \%$ confidence bands around the null

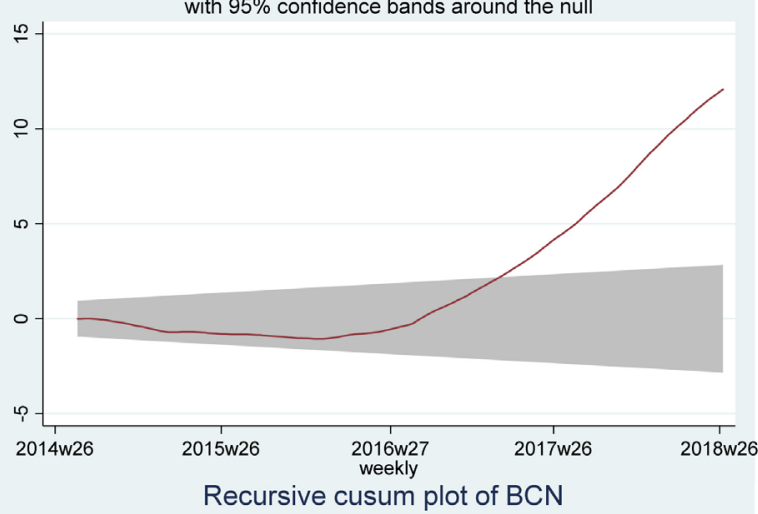

with $95 \%$ confidence bands around the nut

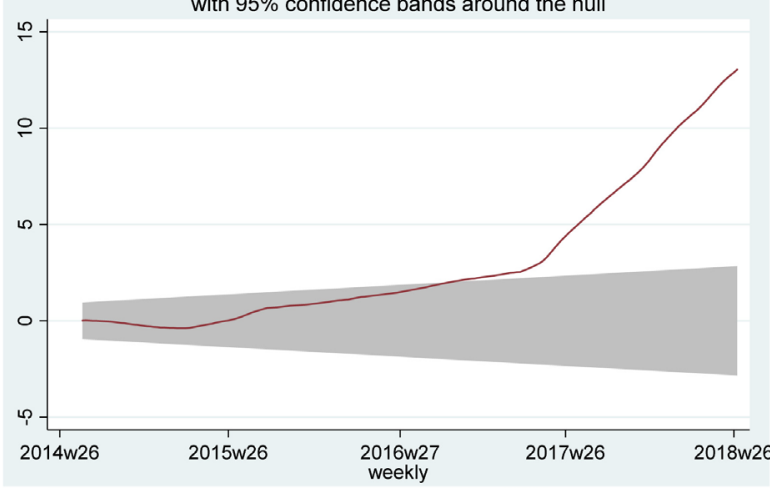

Figure 3. Cusum test for all variables (in $\log$ forms).
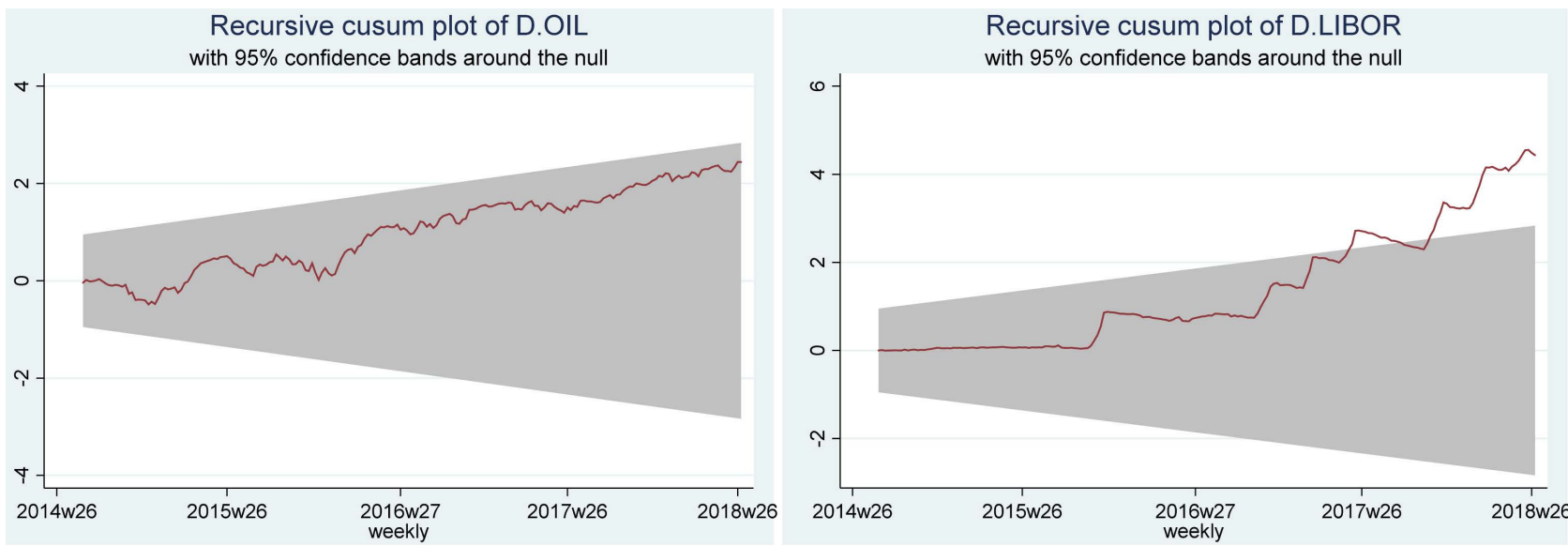


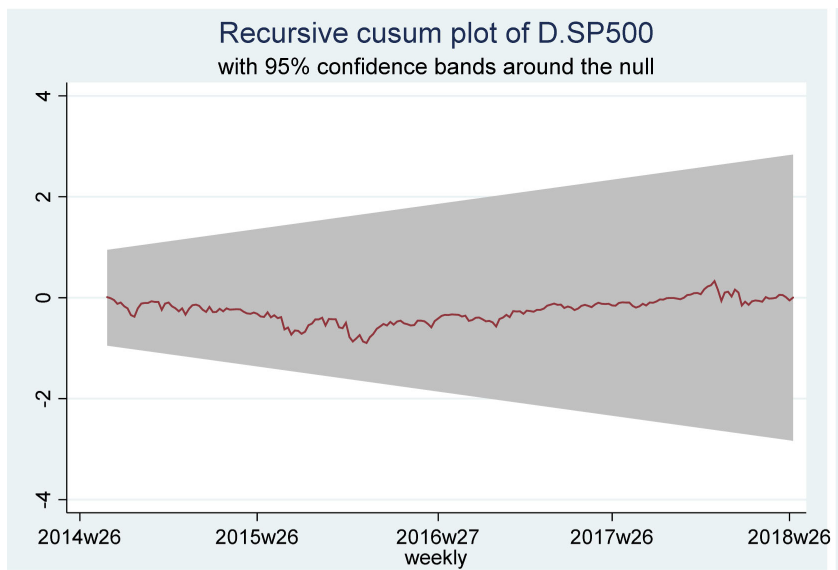

Recursive cusum plot of D.USD

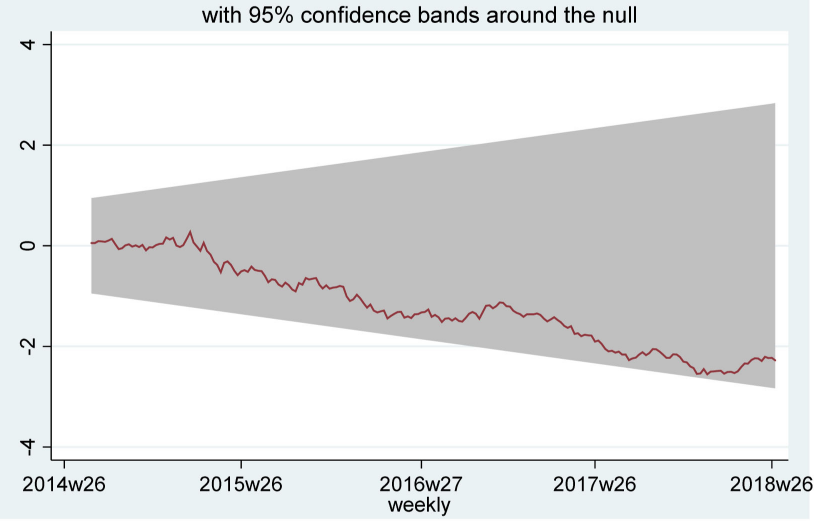

Recursive cusum plot of D.LTC

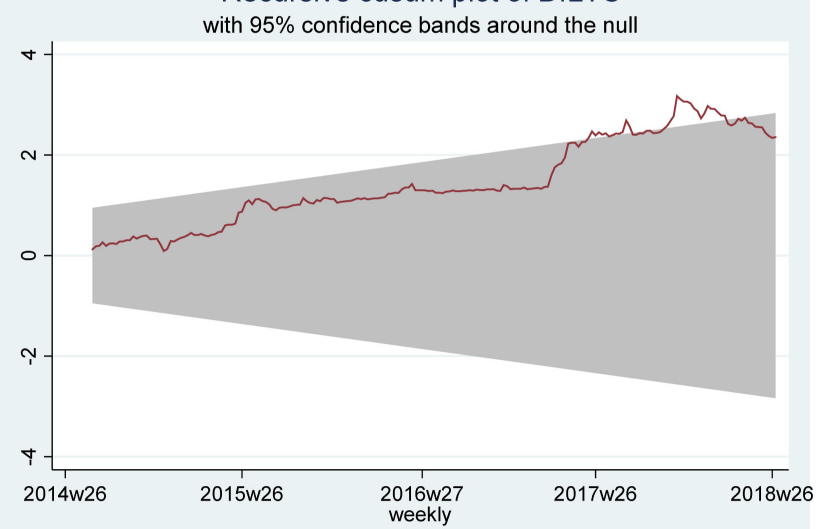

Recursive cusum plot of D.XLM

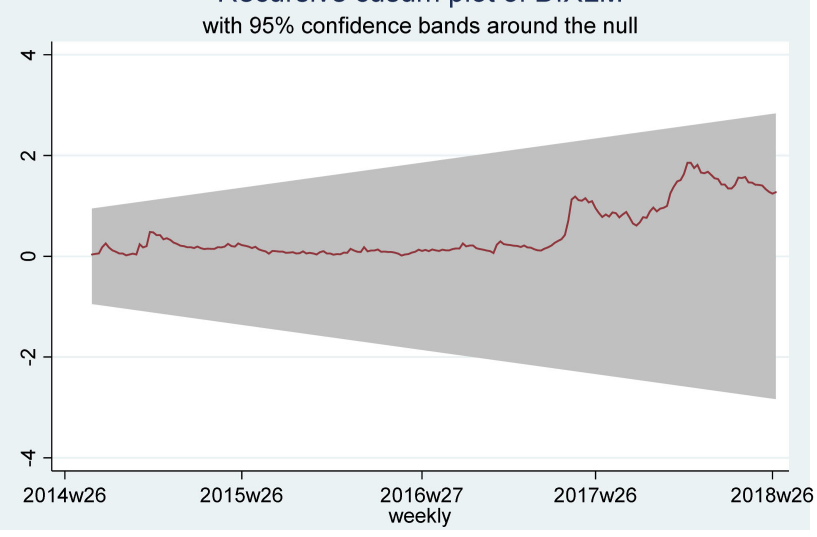

Recursive cusum plot of D.GOLD

with $95 \%$ confidence bands around the null

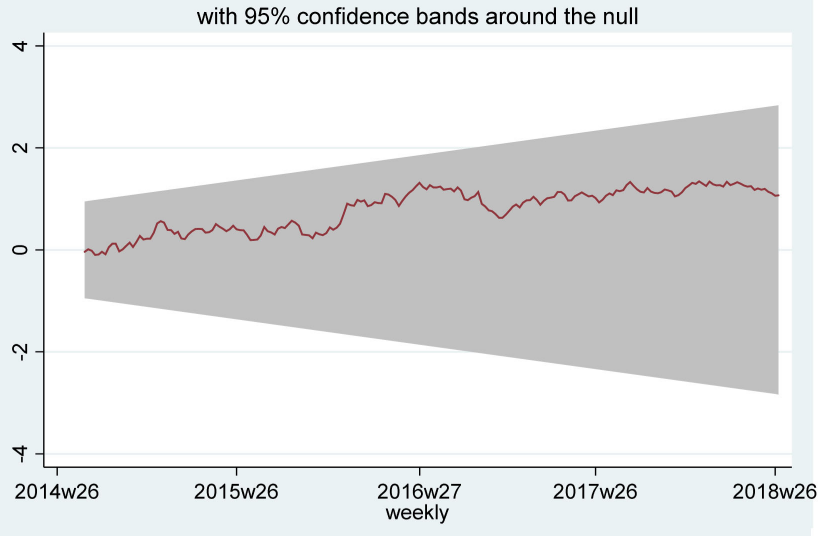

Recursive cusum plot of D.BTC

with $95 \%$ confidence bands around the null

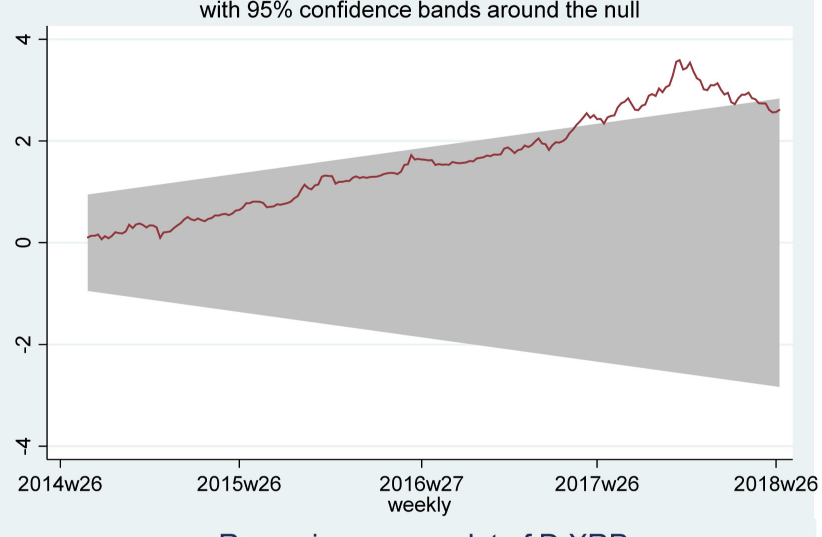

Recursive cusum plot of D.XRP

with $95 \%$ confidence bands around the null

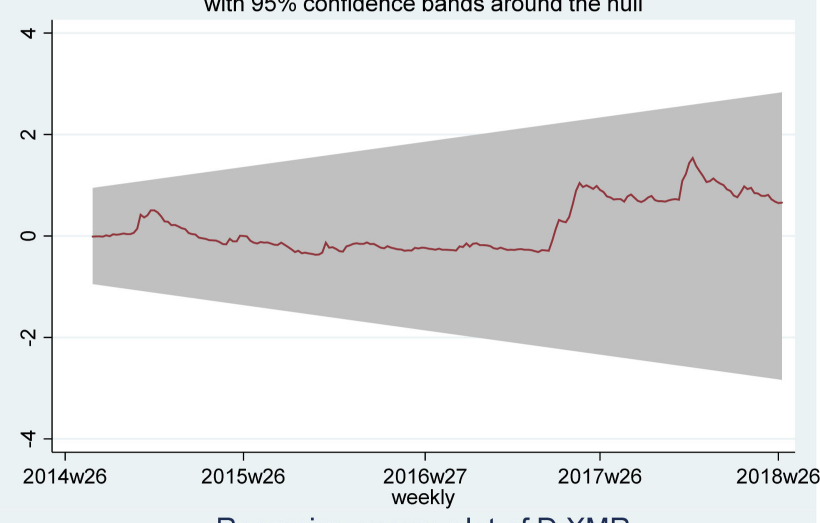

Recursive cusum plot of D.XMR

with $95 \%$ confidence bands around the null

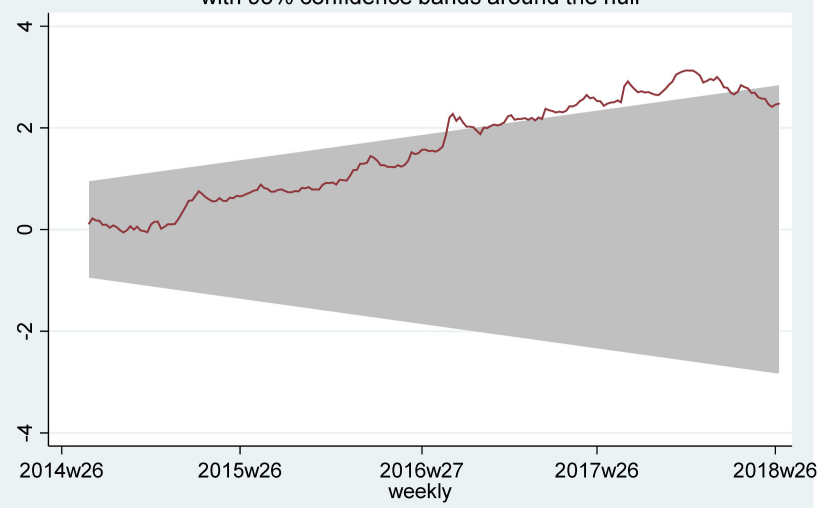




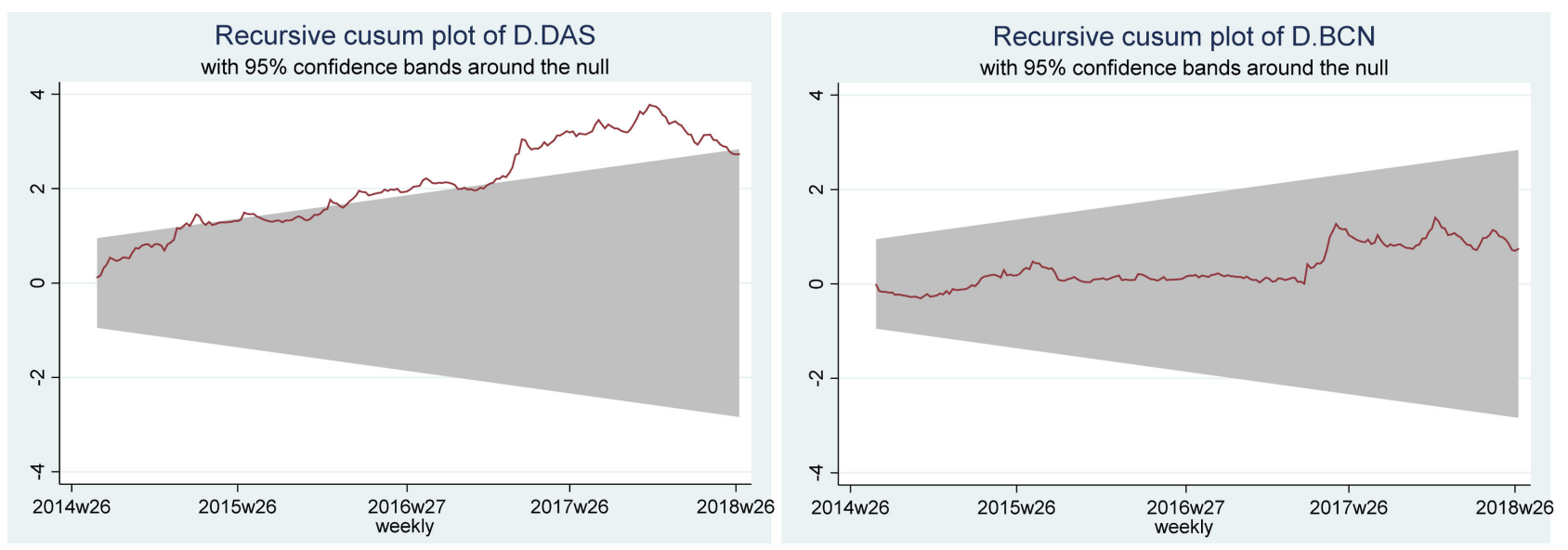

Figure 4. Cusum test for all variables (in 1st difference of $\log$ forms).

Tables 2-4 reports the characteristics of variables examined by the Dicky-Fuller unit root test, Johansen Cointegration test, and Granger causality test, respectively.

Results in Table 2 show that all variables (excluding USD index) have stationary at the $1^{\text {st }}$ difference. For testing cointegration, results in Table 3 show that the LIBOR and the USD index have cointegration with all cryptocurrencies. The S\&P500 index has cointegration with BTC, XRP, DAS and BCN, while the oil price has cointegration with XMR and DAS. Interestingly, the gold price has no cointegration with all cryptocurrencies. These results suggest that there are strong relationships between the USD index and LIBOR with cryptocurrencies.

The results of Granger causality test for each pair of cryptocurrency and economic indicators are presented in Table 4 . The causal relations between these variables are asymmetric. There exists bidirectional causality between the oil price and most of the cryptocurrencies, except for BTC. The USD index causes all cryptocurrencies. However, only BTC, LTC, DAS and BCN, respectively, causes the USD index. The S\&P500 index causes BTC, LTC, and DAS, respectively; and only XRP, XLM, and BCN, respectively causes S\&P500 index. The goldprice causes BTC, XRP, XMR, and DAS, respectively; and only LTC causes the gold price. In summary, the oil price, and the USD index cause most of the selected cryptocurrencies. Conversely, only BTC and LTC are among the cryptocurrencies, which cause the oil price, the USD index, the S\&P500 index and the gold price, respectively.

As in Table 2, all variables are stationary at the $1^{\text {st }}$ difference (excluding USD index. Taking the $1^{\text {st }}$ difference of all variables, we examine the structural breaks and ARCH disturbance for each variable. Results of Table 5 show evidence that there are structural breaks in economic factors (e.g., oil price, LIBOR, USD index). In addition, there is an ARCH disturbance in case of XRP, XLM, XMR and $\mathrm{BCN}$, respectively. We then run GARCH $(1,1)$ for each cryptocurrency with economic factors and results are reported in Table 6.

The results in Table 6 show that there exist structural breaks and ARCH disturbance in the price of each cryptocurrency, suggesting a systematic risk within 
Table 2. Correlation matrix.

\begin{tabular}{|c|c|c|c|c|c|c|c|c|c|c|c|c|}
\hline & OIL & SP500 & GOLD & LIBOR & USD & BTC & XRP & LTC & XLM & XMR & DASH & $\mathrm{BCN}$ \\
\hline OIL & 1 & & & & & & & & & & & \\
\hline SP500 & $\begin{array}{c}0.290^{* * *} \\
(0.000)\end{array}$ & 1 & & & & & & & & & & \\
\hline GOLD & $\begin{array}{c}0.310^{* * *} \\
(0.000)\end{array}$ & $\begin{array}{c}0.556^{\star * *} \\
(0.000)\end{array}$ & 1 & & & & & & & & & \\
\hline LIBOR & $\begin{array}{l}0.221^{\star * *} \\
(0.0015)\end{array}$ & $\begin{array}{c}0.946^{* * *} \\
(0.000)\end{array}$ & $\begin{array}{c}0.563^{\star * *} \\
(0.000)\end{array}$ & 1 & & & & & & & & \\
\hline USD & $\begin{array}{c}-0.703^{\star * *} \\
(0.000)\end{array}$ & $\begin{array}{c}-0.050 \\
(0.4786)\end{array}$ & $\begin{array}{c}-0.375^{\star * *} \\
(0.000)\end{array}$ & $\begin{array}{c}0.067 \\
(0.000)\end{array}$ & 1 & & & & & & & \\
\hline BTC & $\begin{array}{l}0.280^{\star * *} \\
(0.0001)\end{array}$ & $\begin{array}{c}0.944^{\star \star \star} \\
(0.000)\end{array}$ & $\begin{array}{c}0.603^{\star * *} \\
(0.000)\end{array}$ & $\begin{array}{c}0.955^{\star \star \star} \\
(0.000)\end{array}$ & $\begin{array}{c}-0.182^{\star * \star} \\
(0.0091)\end{array}$ & 1 & & & & & & \\
\hline XRP & $\begin{array}{l}0.264^{* * *} \\
(0.0001)\end{array}$ & $\begin{array}{c}0.902^{\star * *} \\
(0.000)\end{array}$ & $\begin{array}{c}0.536^{* * *} \\
(0.000)\end{array}$ & $\begin{array}{c}0.899^{* * *} \\
(0.000)\end{array}$ & $\begin{array}{c}-0.243^{* * *} \\
(0.0005)\end{array}$ & $\begin{array}{c}0.915^{\star * *} \\
(0.000)\end{array}$ & 1 & & & & & \\
\hline LTC & $\begin{array}{c}0.311^{\star * \star} \\
(0.000)\end{array}$ & $\begin{array}{c}0.906^{\star \star \star} \\
(0.000)\end{array}$ & $\begin{array}{c}0.552^{\star * *} \\
(0.000)\end{array}$ & $\begin{array}{c}0.929^{\star * \star} \\
(0.000)\end{array}$ & $\begin{array}{c}-0.291^{\star * \star} \\
(0.000)\end{array}$ & $\begin{array}{c}0.970^{\star * *} \\
(0.000)\end{array}$ & $\begin{array}{c}0.952^{\star * *} \\
(0.000)\end{array}$ & 1 & & & & \\
\hline XLM & $\begin{array}{c}0.365^{\star * *} \\
(0.000)\end{array}$ & $\begin{array}{c}0.883^{\star * *} \\
(0.000)\end{array}$ & $\begin{array}{c}0.511^{\star * *} \\
(0.000)\end{array}$ & $\begin{array}{c}0.889^{* * *} \\
(0.000)\end{array}$ & $\begin{array}{c}-0.301^{\star * \star} \\
(0.000)\end{array}$ & $\begin{array}{c}0.894^{* * *} \\
(0.000)\end{array}$ & $\begin{array}{c}0.967^{\star * *} \\
(0.000)\end{array}$ & $\begin{array}{c}0.934^{* * *} \\
(0.000)\end{array}$ & 1 & & & \\
\hline XMR & $\begin{array}{c}0.282^{\star * *} \\
(0.000)\end{array}$ & $\begin{array}{c}0.943^{\star * \star} \\
(0.000)\end{array}$ & $\begin{array}{c}0.630^{* * *} \\
(0.000)\end{array}$ & $\begin{array}{c}0.944^{\star * *} \\
(0.000)\end{array}$ & $\begin{array}{c}-0.103 \\
(0.1412)\end{array}$ & $\begin{array}{c}0.970^{\star * *} \\
(0.000)\end{array}$ & $\begin{array}{c}0.868^{* * *} \\
(0.000)\end{array}$ & $\begin{array}{c}0.921^{\star * *} \\
(0.000)\end{array}$ & $\begin{array}{c}0.836^{\star * *} \\
(0.000)\end{array}$ & 1 & & \\
\hline DASH & $\begin{array}{c}0.197^{\star * *} \\
(0.005)\end{array}$ & $\begin{array}{c}0.951^{\star * *} \\
(0.000)\end{array}$ & $\begin{array}{c}0.614^{* * *} \\
(0.000)\end{array}$ & $\begin{array}{c}0.949^{* * *} \\
(0.000)\end{array}$ & $\begin{array}{l}-0.089 \\
(0.206)\end{array}$ & $\begin{array}{c}0.975^{\star * *} \\
(0.000)\end{array}$ & $\begin{array}{c}0.911^{\star * *} \\
(0.000)\end{array}$ & $\begin{array}{c}0.944^{* * *} \\
(0.000)\end{array}$ & $\begin{array}{c}0.859^{* * *} \\
(0.000)\end{array}$ & $\begin{array}{c}0.974^{* * *} \\
(0.000)\end{array}$ & 1 & \\
\hline $\mathrm{BCN}$ & $\begin{array}{l}0.176^{* *} \\
(0.012)\end{array}$ & $\begin{array}{c}0.917^{* * *} \\
(0.000)\end{array}$ & $\begin{array}{c}0.529^{* * *} \\
(0.000)\end{array}$ & $\begin{array}{c}0.939^{* * *} \\
(0.000)\end{array}$ & $\begin{array}{c}-0.114 \\
(0.1041)\end{array}$ & $\begin{array}{c}0.944^{* * *} \\
(0.000)\end{array}$ & $\begin{array}{c}0.939^{* * *} \\
(0.000)\end{array}$ & $\begin{array}{c}0.960^{* * *} \\
(0.000)\end{array}$ & $\begin{array}{l}0.91^{\star * *} \\
(0.000)\end{array}$ & $\begin{array}{c}0.923^{* * *} \\
(0.000)\end{array}$ & $\begin{array}{c}0.952^{\star * *} \\
(0.000)\end{array}$ & 1 \\
\hline
\end{tabular}

Note: ${ }^{*}{ }^{* *},{ }^{* *}$ denote significant levels at $10 \%, 5 \%, 1 \%$ respectively. P-values are in parenthesis. All variables are examined in log forms (exclude LIBOR).

Table 3. Dickey Fuller test for stationary for level and first different data.

\begin{tabular}{cccc}
\hline \multirow{2}{*}{ Coin } & \multicolumn{3}{c}{ Dickey Fuller test statistic } \\
\cline { 2 - 4 } & Level Data & $1^{\text {st }}$ Difference Data & Conclusions \\
\hline OIL & -2.358 & $-12.733^{* * *}$ & Stationary at $1^{\text {st }}$ Difference \\
SP500 & -0.681 & $-16.203^{* * *}$ & Stationary at $1^{\text {st }}$ Difference \\
GOLD & -2.378 & $-12.505^{* * *}$ & Stationary at $1^{\text {st }}$ Difference \\
LIBOR & 5.016 & $-6.549^{* * *}$ & Stationary at $1^{\text {st }}$ Difference \\
USD & $-3.330^{* *}$ & - & Stationary at Level \\
BTC & 0.374 & $-12.819^{* * *}$ & Stationary at $1^{\text {st }}$ Difference \\
LTC & 0.020 & $-12.735^{* * *}$ & Stationary at $1^{\text {st }}$ Difference \\
XRP & -0.207 & $-9.872^{* * *}$ & Stationary at $1^{\text {st }}$ Difference \\
XLM & 0.083 & $-11.017^{* * *}$ & Stationary at $1^{\text {st }}$ Difference \\
XMR & 0.213 & $-11.652^{* * *}$ & Stationary at $1^{\text {st }}$ Difference \\
DAS & 0.055 & $-12.073^{* * *}$ & Stationary at $1^{\text {st }}$ Difference \\
BCN & -0.308 & $-12.549^{* * *}$ & Stationary at $1^{\text {st }}$ Difference \\
\hline
\end{tabular}

Note: ${ }^{*}{ }^{* *},{ }^{* *}$ denote significant levels at $10 \%, 5 \%, 1 \%$ respectively. 
Table 4. Johansen Cointegration test.

\begin{tabular}{|c|c|c|c|c|c|c|}
\hline Coin & Asset & Test for rank 0 & $\begin{array}{c}\text { Statistic } \\
\text { value }\end{array}$ & $\begin{array}{l}5 \% \text { critical } \\
\text { value }\end{array}$ & $\begin{array}{c}1 \% \text { critical } \\
\text { value }\end{array}$ & Conclusion \\
\hline \multirow{2}{*}{ BTC } & \multirow{2}{*}{ OIL } & Trace test & 14.425 & 15.41 & 20.04 & No cointegration \\
\hline & & Max eigenvalue & 14.213 & 14.07 & 18.63 & Cointegration \\
\hline \multirow{2}{*}{$\mathrm{XRP}$} & \multirow{2}{*}{ OIL } & Trace test & 10.578 & 15.41 & 20.04 & No cointegration \\
\hline & & Max eigenvalue & 10.576 & 14.07 & 18.63 & No cointegration \\
\hline \multirow{2}{*}{ LTC } & \multirow{2}{*}{ OIL } & Trace test & 11.642 & 15.41 & 20.04 & No cointegration \\
\hline & & Max eigenvalue & 11.490 & 14.07 & 18.63 & No cointegration \\
\hline \multirow{2}{*}{ XLM } & \multirow{2}{*}{ OIL } & Trace test & 11.408 & 15.41 & 20.04 & No cointegration \\
\hline & & Max eigenvalue & 11.397 & 14.07 & 18.63 & No cointegration \\
\hline \multirow{2}{*}{ XMR } & \multirow{2}{*}{ OIL } & Trace test & 24.129 & 15.41 & 20.04 & Cointegration \\
\hline & & Max eigenvalue & 22.818 & 14.07 & 18.63 & Cointegration \\
\hline \multirow{2}{*}{ DAS } & \multirow{2}{*}{ OIL } & Trace test & 19.474 & 15.41 & 20.04 & Cointegration \\
\hline & & Max eigenvalue & 17.786 & 14.07 & 18.63 & Cointegration \\
\hline \multirow{2}{*}{$\mathrm{BCN}$} & \multirow{2}{*}{ OIL } & Trace test & 11.084 & 15.41 & 20.04 & No cointegration \\
\hline & & Max eigenvalue & 10.783 & 14.07 & 18.63 & No cointegration \\
\hline \multirow{2}{*}{ BTC } & \multirow{2}{*}{ SP500 } & Trace test & 17.362 & 15.41 & 20.04 & Cointegration \\
\hline & & Max eigenvalue & 17.062 & 14.07 & 18.63 & Cointegration \\
\hline \multirow{2}{*}{ XRP } & \multirow{2}{*}{ SP500 } & Trace test & 9.391 & 15.41 & 20.04 & No cointegration \\
\hline & & Max eigenvalue & 9.390 & 14.07 & 18.63 & No cointegration \\
\hline \multirow{2}{*}{ LTC } & \multirow{2}{*}{ SP500 } & Trace test & 13.980 & 15.41 & 20.04 & No cointegration \\
\hline & & Max eigenvalue & 13.903 & 14.07 & 18.63 & No cointegration \\
\hline \multirow{2}{*}{ XLM } & \multirow{2}{*}{ SP500 } & Trace test & 11.009 & 15.41 & 20.04 & No cointegration \\
\hline & & Max eigenvalue & 11.007 & 14.07 & 18.63 & No cointegration \\
\hline \multirow{2}{*}{ XMR } & \multirow{2}{*}{ SP500 } & Trace test & 17.480 & 15.41 & 20.04 & No cointegration \\
\hline & & Max eigenvalue & 17.333 & 14.07 & 18.63 & No cointegration \\
\hline \multirow{2}{*}{ DAS } & \multirow{2}{*}{ SP500 } & Trace test & 16.322 & 15.41 & 20.04 & Cointegration \\
\hline & & Max eigenvalue & 16.287 & 14.07 & 18.63 & Cointegration \\
\hline 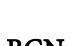 & & Trace test & 10.352 & 15.41 & 20.04 & No cointegration \\
\hline & & Max eigenvalue & 10.346 & 14.07 & 18.63 & No cointegration \\
\hline$P^{n}$ & & Trace test & 11.508 & 15.41 & 20.04 & No cointegration \\
\hline 010 & 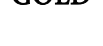 & Max eigenvalue & 11.428 & 14.07 & 18.63 & No cointegration \\
\hline XPP & DISI & Trace test & 9.396 & 15.41 & 20.04 & No cointegration \\
\hline & & Max eigenvalue & 9.341 & 14.07 & 18.63 & No cointegration \\
\hline$T$ & & Trace test & 8.802 & 15.41 & 20.04 & No cointegration \\
\hline & & Max eigenvalue & 8.802 & 14.07 & 18.63 & No cointegration \\
\hline YIM & $\mathrm{CO}$ & Trace test & 8.133 & 15.41 & 20.04 & No cointegration \\
\hline & & Max eigenvalue & 8.125 & 14.07 & 18.63 & No cointegration \\
\hline $\mathrm{YN}$ & & Trace test & 9.876 & 15.41 & 20.04 & No cointegration \\
\hline & & Max eigenvalue & 9.830 & 14.07 & 18.63 & No cointegration \\
\hline DAS & GOLD & Trace test & 11.571 & 15.41 & 20.04 & No cointegration \\
\hline
\end{tabular}


N. P. Canh et al.

\section{Continued}

\begin{tabular}{|c|c|c|c|c|c|c|}
\hline \multirow{3}{*}{$\mathrm{BCN}$} & \multirow{3}{*}{ GOLD } & Max eigenvalue & 11.570 & 14.07 & 18.63 & No cointegration \\
\hline & & Trace test & 8.712 & 15.41 & 20.04 & No cointegration \\
\hline & & Max eigenvalue & 8.622 & 14.07 & 18.63 & No cointegration \\
\hline \multirow{2}{*}{ BTC } & \multirow{2}{*}{ USD } & Trace test & 20.668 & 15.41 & 20.04 & Cointegration \\
\hline & & Max eigenvalue & 20.525 & 14.07 & 18.63 & Cointegration \\
\hline \multirow{2}{*}{ XRP } & \multirow{2}{*}{ USD } & Trace test & 16.059 & 15.41 & 20.04 & Cointegration \\
\hline & & Max eigenvalue & 15.818 & 14.07 & 18.63 & Cointegration \\
\hline \multirow{2}{*}{ LTC } & \multirow{2}{*}{ USD } & Trace test & 21.984 & 15.41 & 20.04 & Cointegration \\
\hline & & Max eigenvalue & 21.561 & 14.07 & 18.63 & Cointegration \\
\hline \multirow{2}{*}{ XLM } & \multirow{2}{*}{ USD } & Trace test & 15.052 & 15.41 & 20.04 & Cointegration \\
\hline & & Max eigenvalue & 15.050 & 14.07 & 18.63 & Cointegration \\
\hline \multirow{2}{*}{ XMR } & \multirow{2}{*}{ USD } & Trace test & 21.245 & 15.41 & 20.04 & Cointegration \\
\hline & & Max eigenvalue & 20.789 & 14.07 & 18.63 & Cointegration \\
\hline \multirow{2}{*}{ DAS } & \multirow{2}{*}{ USD } & Trace test & 28.137 & 15.41 & 20.04 & Cointegration \\
\hline & & Max eigenvalue & 26.304 & 14.07 & 18.63 & Cointegration \\
\hline \multirow{2}{*}{$\mathrm{BCN}$} & \multirow{2}{*}{ USD } & Trace test & 19.157 & 15.41 & 20.04 & Cointegration \\
\hline & & Max eigenvalue & 18.106 & 14.07 & 18.63 & Cointegration \\
\hline \multirow{2}{*}{ BTC } & \multirow{2}{*}{ LIBOR } & Trace test & 32.398 & 15.41 & 20.04 & Cointegration \\
\hline & & Max eigenvalue & 31.709 & 14.07 & 18.63 & Cointegration \\
\hline \multirow{2}{*}{ XRP } & \multirow{2}{*}{ LIBOR } & Trace test & 27.910 & 15.41 & 20.04 & Cointegration \\
\hline & & Max eigenvalue & 25.270 & 14.07 & 18.63 & Cointegration \\
\hline \multirow{2}{*}{ LTC } & \multirow{2}{*}{ LIBOR } & Trace test & 27.685 & 15.41 & 20.04 & Cointegration \\
\hline & & Max eigenvalue & 24.040 & 14.07 & 18.63 & Cointegration \\
\hline \multirow{2}{*}{ XLM } & \multirow{2}{*}{ LIBOR } & Trace test & 29.167 & 15.41 & 20.04 & Cointegration \\
\hline & & Max eigenvalue & 25.314 & 14.07 & 18.63 & Cointegration \\
\hline \multirow{2}{*}{ XMR } & \multirow{2}{*}{ LIBOR } & Trace test & 26.296 & 15.41 & 20.04 & Cointegration \\
\hline & & Max eigenvalue & 25.771 & 14.07 & 18.63 & Cointegration \\
\hline \multirow{2}{*}{ DAS } & \multirow{2}{*}{ LIBOR } & Trace test & 25.597 & 15.41 & 20.04 & Cointegration \\
\hline & & Max eigenvalue & 25.592 & 14.07 & 18.63 & Cointegration \\
\hline \multirow{2}{*}{$\mathrm{BCN}$} & \multirow{2}{*}{ LIBOR } & Trace test & 28.396 & 15.41 & 20.04 & Cointegration \\
\hline & & Max eigenvalue & 24.308 & 14.07 & 18.63 & Cointegration \\
\hline
\end{tabular}

Note: ${ }^{* *},{ }^{* *}$ denote significant levels at $10 \%, 5 \%, 1 \%$ respectively. All of pair asset are tested to obtain suitable lag-order selection statistics.

Table 5. Granger causality tests for each of pair assets.

\begin{tabular}{|c|c|c|c|c|c|c|c|c|c|c|c|c|c|c|}
\hline Equation & Excluded & Chi2 & $\mathrm{df}$ & P-value & Equation & Excluded & Chi2 & $\mathrm{df}$ & P-Value & Equation & Excluded & Chi2 & $\mathrm{df}$ & P-Value \\
\hline BTC & OIL & $2.903^{*}$ & 1 & 0.088 & BTC & SP500 & 2.170 & 1 & 0.141 & BTC & GOLD & 1.288 & 2 & 0.525 \\
\hline OIL & BTC & $5.968^{* *}$ & 1 & 0.015 & SP500 & BTC & $14.353^{* * *}$ & 1 & 0.000 & GOLD & BTC & $6.48^{* *}$ & 2 & 0.039 \\
\hline $\mathrm{XRP}$ & OIL & 0.383 & 2 & 0.826 & XRP & SP500 & $7.826^{* *}$ & 2 & 0.020 & XRP & GOLD & 0.101 & 2 & 0.951 \\
\hline OIL & XRP & $4.935^{*}$ & 2 & 0.085 & SP500 & XRP & 3.172 & 2 & 0.205 & GOLD & XRP & $6.957^{* *}$ & 2 & 0.031 \\
\hline
\end{tabular}


N. P. Canh et al.

Continued

\begin{tabular}{|c|c|c|c|c|c|c|c|c|c|c|c|c|c|c|}
\hline LTC & OIL & 1.012 & 1 & 0.315 & LTC & SP500 & $7.115^{* *}$ & 2 & 0.029 & LTC & GOLD & $5.166^{*}$ & 2 & 0.076 \\
\hline OIL & LTC & $4.948^{* *}$ & 1 & 0.026 & SP500 & LTC & $5.781^{*}$ & 2 & 0.056 & GOLD & LTC & 4.065 & 2 & 0.131 \\
\hline XLM & OIL & 0.371 & 2 & 0.831 & XLM & SP500 & $9.238^{* *}$ & 2 & 0.010 & XLM & GOLD & 0.739 & 2 & 0.691 \\
\hline OIL & XLM & $7.517^{\star *}$ & 2 & 0.023 & SP500 & XLM & 2.377 & 2 & 0.305 & GOLD & XLM & 3.582 & 2 & 0.167 \\
\hline XMR & OIL & 3.820 & 2 & 0.148 & XMR & SP500 & 2.103 & 2 & 0.349 & $\mathrm{XMR}$ & GOLD & 1.647 & 2 & 0.439 \\
\hline OIL & XMR & $8.003^{* *}$ & 2 & 0.018 & SP500 & XMR & $15.51^{* * *}$ & 2 & 0.000 & GOLD & XMR & $6.013^{* *}$ & 2 & 0.049 \\
\hline DAS & OIL & 2.764 & 2 & 0.251 & DAS & SP500 & 1.949 & 2 & 0.377 & DAS & GOLD & 2.443 & 2 & 0.295 \\
\hline OIL & DAS & $7.047^{\star *}$ & 2 & 0.03 & SP500 & DAS & $11.968^{* * *}$ & 2 & 0.003 & GOLD & DAS & $8.412^{\star *}$ & 2 & 0.015 \\
\hline $\mathrm{BCN}$ & OIL & 0.269 & 1 & 0.604 & $\mathrm{BCN}$ & SP500 & $9.228^{\star *}$ & 2 & 0.010 & $\mathrm{BCN}$ & GOLD & 0.859 & 2 & 0.651 \\
\hline OIL & $\mathrm{BCN}$ & $5.301^{* *}$ & 1 & 0.021 & SP500 & $\mathrm{BCN}$ & 4.434 & 2 & 0.109 & GOLD & $\mathrm{BCN}$ & 3.860 & 2 & 0.145 \\
\hline BTC & USD & $6.263^{\star *}$ & 1 & 0.012 & BTC & LIBOR & 1.413 & 2 & 0.493 & & & & & \\
\hline USD & BTC & $3.636^{*}$ & 1 & 0.057 & LIBOR & BTC & $10.007^{* * *}$ & 2 & 0.007 & & & & & \\
\hline XRP & USD & 0.431 & 2 & 0.806 & $\mathrm{XRP}$ & LIBOR & $8.350^{* *}$ & 2 & 0.015 & & & & & \\
\hline USD & $\mathrm{XRP}$ & $5.462^{*}$ & 2 & 0.065 & LIBOR & $\mathrm{XRP}$ & 1.482 & 2 & 0.477 & & & & & \\
\hline LTC & USD & $6.677^{* *}$ & 1 & 0.010 & LTC & LIBOR & 4.019 & 2 & 0.134 & & & & & \\
\hline USD & LTC & $5.389^{* *}$ & 1 & 0.020 & LIBOR & LTC & 1.286 & 2 & 0.526 & & & & & \\
\hline XLM & USD & 0.335 & 1 & 0.563 & XLM & LIBOR & $9.360^{*}$ & 4 & 0.053 & & & & & \\
\hline USD & XLM & $3.905^{* *}$ & 1 & 0.048 & LIBOR & XLM & 6.397 & 4 & 0.171 & & & & & \\
\hline XMR & USD & 3.947 & 2 & 0.139 & XMR & LIBOR & 1.364 & 2 & 0.506 & & & & & \\
\hline USD & XMR & $4.797^{\star}$ & 2 & 0.091 & LIBOR & XMR & 2.797 & 2 & 0.247 & & & & & \\
\hline DAS & USD & $17.358^{\star * *}$ & 1 & 0.000 & DAS & LIBOR & 0.330 & 2 & 0.848 & & & & & \\
\hline USD & DAS & $4.282^{* *}$ & 1 & 0.039 & LIBOR & DAS & 1.779 & 2 & 0.411 & & & & & \\
\hline $\mathrm{BCN}$ & USD & $3.149^{*}$ & 1 & 0.076 & $\mathrm{BCN}$ & LIBOR & $25.452^{* * *}$ & 4 & 0.000 & & & & & \\
\hline USD & BCN & $5.196^{* *}$ & 1 & 0.023 & LIBOR & $\mathrm{BCN}$ & $9.481^{*}$ & 4 & 0.050 & & & & & \\
\hline
\end{tabular}

Note: ${ }^{*}{ }^{* *},{ }^{* *}$ denote significant levels at $10 \%, 5 \%, 1 \%$ respectively. All of pair asset are tested to obtain suitable lag-order selection statistics.

Table 6. Cumulative sum test and Structural Break test for 1st Difference Data.

\begin{tabular}{|c|c|c|c|c|c|c|c|c|c|c|c|}
\hline \multirow[t]{2}{*}{ Coin } & \multicolumn{5}{|c|}{ Cumulative sum test for parameter stability } & \multicolumn{3}{|c|}{ Structural break test } & \multicolumn{3}{|c|}{$\begin{array}{l}\text { LM test for autoregressive } \\
\text { conditional heteroskedasticity } \\
\text { [ARCH] }\end{array}$} \\
\hline & $\begin{array}{c}\text { Test } \\
\text { statistic }\end{array}$ & $\begin{array}{c}1 \% \text { critical } \\
\text { value }\end{array}$ & $\begin{array}{c}5 \% \text { critical } \\
\text { value }\end{array}$ & $\begin{array}{l}10 \% \text { critical } \\
\text { value }\end{array}$ & Conclusions & Swald test & $\begin{array}{c}\text { Estimated } \\
\text { break week }\end{array}$ & Conclusions & Chi2 & p-value & Conclusion \\
\hline D.OIL & 0.818 & 1.1430 & 0.9479 & 0.850 & No Break & $11.660^{* *}$ & $2016 w 3$ & Break & 0.847 & 0.3573 & $\begin{array}{c}\text { No ARCH } \\
\text { effects }\end{array}$ \\
\hline D.LIBOR & $1.529^{* * *}$ & 1.1430 & 0.9479 & 0.850 & Break & $34.769^{\star \star \star}$ & $2016 w 46$ & Break & $28.874^{\star * *}$ & 0.0000 & $\begin{array}{c}\text { ARCH[p] } \\
\text { disturbance }\end{array}$ \\
\hline D.SP500 & 0.509 & 1.1430 & 0.9479 & 0.850 & No Break & 2.061 & 2016w7 & No Break & $4.426^{\star *}$ & 0.0354 & $\begin{array}{c}\mathrm{ARCH}[\mathrm{p}] \\
\text { disturbance }\end{array}$ \\
\hline D.GOLD & 0.667 & 1.1430 & 0.9479 & 0.850 & No Break & 2.494 & $2015 w 49$ & No Break & 0.067 & 0.7965 & $\begin{array}{l}\text { No ARCH } \\
\text { effects }\end{array}$ \\
\hline
\end{tabular}




\begin{tabular}{|c|c|c|c|c|c|c|c|c|c|c|c|}
\hline \multicolumn{12}{|c|}{ Continued } \\
\hline D.USD & $0.919^{*}$ & 1.1430 & 0.9479 & 0.850 & Break & $12.467^{* * *}$ & $2015 w 12$ & Break & 1.508 & 0.2195 & $\begin{array}{c}\text { No ARCH } \\
\text { effects }\end{array}$ \\
\hline D.BTC & $1.323^{* * *}$ & 1.1430 & 0.9479 & 0.850 & Break & 6.608 & $2015 w 17$ & No Break & 1.877 & 0.1707 & $\begin{array}{c}\text { No ARCH } \\
\text { effects }\end{array}$ \\
\hline D.LTC & $1.170^{* * *}$ & 1.1430 & 0.9479 & 0.850 & Break & 6.181 & $2015 w 17$ & No Break & 0.831 & 0.3621 & $\begin{array}{c}\text { No ARCH } \\
\text { effects }\end{array}$ \\
\hline D.XRP & 0.561 & 1.1430 & 0.9479 & 0.850 & No Break & 4.466 & $2017 w 12$ & No Break & $15.396^{* * *}$ & 0.0001 & $\begin{array}{c}\text { ARCH[p] } \\
\text { disturbance }\end{array}$ \\
\hline D.XLM & 0.677 & 1.1430 & 0.9479 & 0.850 & No Break & 5.196 & $2017 w 11$ & No Break & $13.810^{* * *}$ & 0.0002 & $\begin{array}{c}\mathrm{ARCH}[\mathrm{p}] \\
\text { disturbance }\end{array}$ \\
\hline D.XMR & $1.146^{* * *}$ & 1.1430 & 0.9479 & 0.850 & Break & 6.728 & $2015 w 50$ & No Break & $5.156^{\star *}$ & 0.023 & $\begin{array}{c}\text { ARCH[p] } \\
\text { disturbance }\end{array}$ \\
\hline D.DAS & $1.387^{* * *}$ & 1.1430 & 0.9479 & 0.850 & Break & 5.142 & $2017 w 49$ & No Break & 0.000 & 1.0000 & $\begin{array}{c}\text { No ARCH } \\
\text { effects }\end{array}$ \\
\hline D.BCN & 0.522 & 1.1430 & 0.9479 & 0.850 & No Break & 2.794 & $2017 w 13$ & No Break & $3.026^{*}$ & 0.0819 & $\begin{array}{c}\mathrm{ARCH}[\mathrm{p}] \\
\text { disturbance }\end{array}$ \\
\hline
\end{tabular}

Note: ${ }^{* *},{ }^{* * *}$ denote significant levels at $10 \%, 5 \%, 1 \%$ respectively.

cryptocurrency markets. Concerning economic factors, observations show that the USD index has negative effects on all seven cryptocurrencies, while other economic factors have inconsistent effects on all cryptocurrencies. The implication drawn from these results is that cryptocurrencies are considered as a financial asset to hedge systematic risk from economic factors.

\subsection{Check Robustness}

The inconsistent results of economic factors in line with the existence of structural breaks and ARCH disturbance among variables leading to an ideal condition for DCC MGARCH model in which the conditional correlation matrix from estimation is robust to analyse the relationship among variables [30] [31]. All results from DCC MGARCH are reported in Tables 7-13 for each cryptocurrency.

For BTC, as in Table 7 the oil price, the S\&P500 index, and LIBOR have significantly negative correlations with BTC. The results suggest that BTC seems to not be a tool for hedging the risk of USD index and gold price. Our finding is different from the studies [20] [21] that Bitcoin can hedge against USD or any currency.

For XRP, the results of Table 8 show that XRP has a significant negative correlation with the oil price. Moreover, as in Table 4, the oil price causes XRP. These results suggest that the increased oil price reduces the price of XRP.

For other cryptocurrencies, as in Tables 9-14 DAS has a positive correlation with LIBOR, but negative correlation with USD index. XLM has a positive correlation with SP500 index. Our findings show that the correlations between cryptocurrencies and economic factors are inconsistent, suggesting that cryptocurrencies may be not tools or financial assets to hedge systematic risks, which are caused by economic factors. 
Table 7. GARCH $(1,1)$ for each cryptocurrency.

\begin{tabular}{|c|c|c|c|c|c|c|c|}
\hline Variables & BTC & XRP & LTC & XLM & XMR & DAS & $\mathrm{BCN}$ \\
\hline \multirow{2}{*}{ OIL } & 0.013 & $-0.467^{\star \star \star}$ & 0.307 & -0.0004 & -0.313 & 0.043 & 0.130 \\
\hline & {$[0.146]$} & [0.149] & {$[0.229]$} & {$[0.209]$} & {$[0.325]$} & [0.197] & {$[0.339]$} \\
\hline \multirow{2}{*}{ SP500 } & 0.092 & $1.560^{* * *}$ & -0.773 & 0.934 & -0.110 & 0.330 & -0.647 \\
\hline & {$[0.426]$} & [0.483] & [0.505] & [0.945] & {$[0.844]$} & {$[0.551]$} & {$[0.822]$} \\
\hline \multirow{2}{*}{ GOLD } & -0.347 & 0.670 & -0.093 & 0.353 & -0.280 & -0.225 & 0.603 \\
\hline & 0.405 & [0.481] & [0.629] & {$[0.765]$} & {$[0.806]$} & [0.689] & {$[0.872]$} \\
\hline \multirow{2}{*}{ USD } & -0.555 & $-1.285^{\star \star}$ & -1.497 & -0.338 & -1.619 & $-2.596^{\star *}$ & 1.119 \\
\hline & [0.893] & [0.653] & [1.041] & {$[1.775]$} & [1.488] & {$[1.062]$} & [1.314] \\
\hline \multirow[t]{2}{*}{ LIBOR } & -0.048 & $4.342^{* * *}$ & 0.105 & 0.323 & $1.177^{*}$ & $1.454^{\star *}$ & $-3.292^{* * *}$ \\
\hline & [0.447] & [0.380] & [0.462] & {$[0.635]$} & {$[0.601]$} & [0.645] & {$[0.704]$} \\
\hline \multirow[t]{2}{*}{ cons } & 0.009 & $-0.025^{\star \star}$ & 0.001 & 0.0002 & 0.008 & 0.002 & $0.036^{*}$ \\
\hline & [0.013] & {$[0.01]$} & {$[0.012]$} & {$[0.014]$} & {$[0.020]$} & {$[0.016]$} & [0.021] \\
\hline \multicolumn{8}{|l|}{ ARMA } \\
\hline \multirow[t]{2}{*}{$\operatorname{AR}(1)$} & $0.969^{* * *}$ & -0.308 & $-0.844^{\star * \star}$ & $-0.911^{\star * *}$ & 0.511 & 0.483 & 0.457 \\
\hline & [0.161] & [0.286] & [0.131] & [0.094] & {$[0.618]$} & {$[0.380]$} & {$[0.314]$} \\
\hline \multirow[t]{2}{*}{$\mathrm{MA}(1)$} & $-0.860^{\star * *}$ & $0.554^{* *}$ & $0.861^{\star * *}$ & $1.064^{* * *}$ & -0.355 & -0.283 & -0.229 \\
\hline & [0.188] & [0.241] & [0.131] & [0.106] & {$[0.674]$} & {$[0.416]$} & [0.299] \\
\hline \multirow[t]{2}{*}{ MA(3) } & -0.090 & $-0.115^{\star \star}$ & $-0.096^{\star \star}$ & $-0.192^{\star * *}$ & -0.034 & 0.009 & 0.069 \\
\hline & {$[0.080]$} & {$[0.048]$} & {$[0.046]$} & {$[0.048]$} & [0.109] & [0.095] & {$[0.097]$} \\
\hline \multicolumn{8}{|l|}{ ARCH } \\
\hline \multirow[t]{2}{*}{ L1.ARCH } & $0.157^{\star *}$ & $1.139^{* * *}$ & $0.699^{* * *}$ & $0.347^{\star * *}$ & $0.213^{\star *}$ & $0.236^{\star *}$ & $0.650^{* * *}$ \\
\hline & {$[0.07]$} & [0.186] & [0.145] & [0.083] & {$[0.100]$} & [0.115] & {$[0.204]$} \\
\hline \multirow[t]{2}{*}{ L1.GARCH } & $0.788^{\star * *}$ & $0.212^{* * *}$ & 0.055 & $0.614^{\star * \star}$ & 0.202 & $0.533^{\star * \star}$ & $0.303^{\star *}$ \\
\hline & {$[0.084]$} & {$[0.072]$} & {$[0.065]$} & {$[0.074]$} & {$[0.304]$} & {$[0.200]$} & {$[0.124]$} \\
\hline \multirow[t]{2}{*}{ cons GARCH } & $0.001^{*}$ & $0.004^{\star *}$ & $0.011^{\star \star \star}$ & $0.004^{\star * \star}$ & $0.019^{\star *}$ & $0.005^{\star}$ & $0.014^{* * *}$ \\
\hline & {$[0.0003]$} & {$[0.002]$} & {$[0.001]$} & {$[0.002]$} & {$[0.009]$} & {$[0.003]$} & {$[0.005]$} \\
\hline $\mathrm{N}$ & 203 & 203 & 203 & 203 & 203 & 203 & 203 \\
\hline Log likelihood & 192.008 & 81.109 & 107.206 & 54.210 & 66.557 & 112.707 & 18.956 \\
\hline Chi2 & $266.4^{* * *}$ & $284.88^{* * *}$ & $122.06^{* * *}$ & $128.88^{* * *}$ & $16.38^{* *}$ & $34.89^{* * *}$ & $39.91^{* * *}$ \\
\hline \multicolumn{8}{|c|}{ Test L1.ARCH $=0$; L1.GARCH $=0$} \\
\hline Chi2[2] & $531.07^{\star \star \star}$ & $70.35^{\star * \star}$ & $31.17^{\star \star \star}$ & $189.11^{\star * *}$ & $6.39^{* *}$ & $41.47^{\star * *}$ & $43.89^{\star * *}$ \\
\hline
\end{tabular}

Note: ${ }^{*}, * *, * *$ denote significant levels at $10 \%, 5 \%, 1 \%$ respectively. Standard errors are in bracket.

Table 8. Dynamic conditional correlation MGARCH model of Bitcoin.

\begin{tabular}{ccccccc}
\hline Variables & BTC & OIL & SP500 & GOLD & USD & LIBOR \\
\hline \multirow{2}{*}{ Cons } & 0.009 & 0.0002 & $0.002^{* *}$ & 0.0001 & 0.0006 & $0.003^{* * *}$ \\
& {$[0.006]$} & {$[0.003]$} & {$[0.001]$} & {$[0.001]$} & {$[0.0007]$} & {$[0.0007]$} \\
\hline
\end{tabular}




\section{Continued}

\begin{tabular}{|c|c|c|c|c|c|c|}
\hline L1.ARCH & $\begin{array}{l}0.168^{* *} \\
{[0.071]}\end{array}$ & $\begin{array}{c}0.084^{*} \\
{[0.051]}\end{array}$ & $\begin{array}{c}0.227^{* * *} \\
{[0.072]}\end{array}$ & $\begin{array}{c}0.060 \\
{[0.048]}\end{array}$ & $\begin{array}{c}0.07^{\star} \\
{[0.0403]}\end{array}$ & $\begin{array}{c}0.957^{* * *} \\
{[0.180]}\end{array}$ \\
\hline L1.GARCH & $\begin{array}{c}0.776^{\star * *} \\
{[0.071]}\end{array}$ & $\begin{array}{c}0.786^{\star * \star} \\
{[0.123]}\end{array}$ & $\begin{array}{c}0.712^{* * *} \\
{[0.072]}\end{array}$ & $\begin{array}{c}-0.798^{\star * *} \\
{[0.227]}\end{array}$ & $\begin{array}{c}0.881^{* * *} \\
{[0.087]}\end{array}$ & $\begin{array}{l}-0.005 \\
{[0009]}\end{array}$ \\
\hline Cons ARCH & $\begin{array}{c}0.001^{* *} \\
{[0.0003]}\end{array}$ & $\begin{array}{c}0.0003 \\
{[0.0002]}\end{array}$ & $\begin{array}{c}0.00002^{*} \\
{[0.00001]}\end{array}$ & $\begin{array}{l}0.006^{* * *} \\
{[0.0001]}\end{array}$ & $\begin{array}{c}0.000005 \\
{[0.000007]}\end{array}$ & $\begin{array}{l}0.00006^{* * *} \\
{[0.000008]}\end{array}$ \\
\hline $\mathrm{N}$ & \multicolumn{6}{|l|}{203} \\
\hline Log likelihood & \multicolumn{6}{|l|}{2905.324} \\
\hline Chi2 & $5.52^{*}$ & & & & & \\
\hline Lambda1 & $\begin{array}{c}0.0402 \\
{[0.025]}\end{array}$ & & & & & \\
\hline Lambda2 & $\begin{array}{c}0.330 \\
{[0.278]}\end{array}$ & & & & & \\
\hline Correlations & BTC & & IL & SP500 & GOLD & USD \\
\hline OIL & $\begin{array}{c}0.002 \\
{[0.074]}\end{array}$ & & & & & \\
\hline SP500 & $\begin{array}{c}0.050 \\
{[0.074]}\end{array}$ & & $\begin{array}{l}2^{* * *} \\
73]\end{array}$ & & & \\
\hline GOLD & $\begin{array}{l}-0.015 \\
{[0.075]}\end{array}$ & & $\begin{array}{l}39 \\
75]\end{array}$ & $\begin{array}{l}-0.189^{\star * *} \\
{[0.072]}\end{array}$ & & \\
\hline USD & $\begin{array}{l}-0.048 \\
{[0.075]}\end{array}$ & & $\begin{array}{l}115 \\
74]\end{array}$ & $\begin{array}{c}0.139^{\star} \\
{[0.075]}\end{array}$ & $\begin{array}{c}0.556^{\star * *} \\
{[0.052]}\end{array}$ & \\
\hline LIBOR & $\begin{array}{c}0.076 \\
{[0.075]}\end{array}$ & & $\begin{array}{l}01 \\
76]\end{array}$ & $\begin{array}{l}-0.019 \\
{[0.075]}\end{array}$ & $\begin{array}{l}-0.117 \\
{[0.074]}\end{array}$ & $\begin{array}{l}-0.014 \\
{[0.075]}\end{array}$ \\
\hline
\end{tabular}

Note: ${ }^{* *}$ and ${ }^{* *}$ denote the significance level at $10 \%, 5 \%$ and $1 \%$. Standard errors are in bracket.

Table 9. Dynamic conditional correlation MGARCH model of Ripple.

\begin{tabular}{|c|c|c|c|c|c|c|}
\hline Variables & XRP & OIL & SP500 & GOLD & USD & LIBOR \\
\hline \multirow{2}{*}{ Cons } & -0.006 & 0.001 & $0.002^{\star *}$ & -0.00005 & 0.001 & $0.003^{\star * *}$ \\
\hline & {$[0.012]$} & {$[0.003]$} & {$[0.001]$} & {$[0.001]$} & {$[0.001]$} & {$[0.001]$} \\
\hline \multirow{2}{*}{ L1.ARCH } & $0.484^{* * *}$ & $0.086^{\star}$ & $0.216^{\star * *}$ & 0.064 & $0.069^{*}$ & $0.956^{\star * *}$ \\
\hline & {$[0.146]$} & {$[0.052]$} & {$[0.070]$} & {$[0.056]$} & [0.039] & {$[0.180]$} \\
\hline \multirow{2}{*}{ L1.GARCH } & -0.010 & $0.792^{\star * *}$ & $0.721^{\star * *}$ & $-0.770^{\star *}$ & $0.879^{* * *}$ & -0.005 \\
\hline & {$[0.068]$} & [0.124] & {$[0.072]$} & [0.299] & [0.087] & [0.009] \\
\hline \multirow{2}{*}{ Cons ARCH } & $0.024^{* * *}$ & 0.0002 & $0.00002^{*}$ & $0.001^{\star * *}$ & 0.00006 & $0.00006^{* * *}$ \\
\hline & {$[0.003]$} & {$[0.0002]$} & {$[0.00001]$} & {$[0.0001]$} & {$[0.00007]$} & [0.000008] \\
\hline \multirow{3}{*}{ Log likelihood } & 203 & & & & & \\
\hline & 2776.066 & & & & & \\
\hline & \multicolumn{6}{|c|}{ Test $\mathrm{b}[$ Adjustment:lambda1 $]=\mathrm{b}[$ Adjustment:lambda2 $]=0$} \\
\hline Chi2 & 3.07 & & & & & \\
\hline \multirow{2}{*}{ Lambda1 } & 0.033 & & & & & \\
\hline & [0.023] & & & & & \\
\hline
\end{tabular}




\section{Continued}

\begin{tabular}{cccccc}
\hline Lambda2 & 0.264 & & & & \\
& {$[0.361]$} & & & & \\
\hline Correlations & XRP & OIL & SP500 & GOLD & USD \\
\hline OIL & $-0.133^{*}$ & & & & \\
& {$[0.072]$} & & & & \\
SP500 & 0.042 & $0.221^{* * *}$ & & & \\
& {$[0.073]$} & {$[0.071]$} & & & \\
GOLD & 0.038 & 0.041 & $-0.195^{* * *}$ & & \\
& {$[0.074]$} & {$[0.075]$} & {$[0.071]$} & & \\
USD & -0.076 & -0.108 & $0.143^{*}$ & $-0.560^{* * *}$ & \\
& {$[0.074]$} & {$[0.074]$} & {$[0.074]$} & {$[0.052]$} & -0.015 \\
LIBOR & 0.054 & 0.006 & -0.014 & -0.115 & {$[0.074]$} \\
\hline
\end{tabular}

Note: ${ }^{*}{ }^{* *}$ and ${ }^{* *}$ denote the significance level at $10 \%, 5 \%$ and $1 \%$. Standard errors are in bracket.

Table 10. Dynamic conditional correlation MGARCH model of Litecoin.

\begin{tabular}{|c|c|c|c|c|c|c|}
\hline Variables & LTC & OIL & SP500 & GOLD & USD & LIBOR \\
\hline \multirow{2}{*}{ Cons } & -0.003 & 0.0002 & $0.002^{\star *}$ & 0.0001 & 0.001 & $0.003^{* * *}$ \\
\hline & {$[0.010]$} & {$[0.003]$} & {$[0.001]$} & {$[0.001]$} & {$[0.001]$} & {$[0.001]$} \\
\hline \multirow{2}{*}{ L1.ARCH } & $0.457^{\star * *}$ & $0.085^{*}$ & $0.237^{\star * *}$ & 0.063 & $0.074^{*}$ & $0.950^{* * *}$ \\
\hline & {$[0.170]$} & {$[0.051]$} & {$[0.074]$} & {$[0.049]$} & {$[0.040]$} & {$[0.179]$} \\
\hline \multirow{2}{*}{ L1.GARCH } & 0.181 & $0.788^{\star * *}$ & $0.707^{\star * *}$ & $-0.792^{* * *}$ & $0.876^{\star * *}$ & -0.005 \\
\hline & {$[0.168]$} & {$[0.122]$} & {$[0.072]$} & {$[0.238]$} & {$[0.084]$} & [0.009] \\
\hline \multirow{2}{*}{ Cons ARCH } & $0.011^{\star * *}$ & 0.0003 & $0.00002^{*}$ & $0.001^{* * *}$ & 0.00001 & $0.00006^{* * *}$ \\
\hline & {$[0.003]$} & {$[0.0002]$} & {$[0.00001]$} & {$[0.0001]$} & {$[0.00001]$} & {$[0.00001]$} \\
\hline \multirow{3}{*}{ Log likelihood } & 203 & & & & & \\
\hline & 2820.564 & & & & & \\
\hline & \multicolumn{5}{|c|}{ Test $\mathrm{b}[$ Adjustment:lambda1 $]=\mathrm{b}[$ Adjustment:lambda2 $]=0$} & \\
\hline Chi2 & $6.41^{\star *}$ & & & & & \\
\hline \multirow{2}{*}{ Lambda1 } & $0.05^{\star}$ & & & & & \\
\hline & {$[0.026]$} & & & & & \\
\hline \multirow{2}{*}{ Lambda2 } & 0.295 & & & & & \\
\hline & {$[0.274]$} & & & & & \\
\hline Correlations & LTC & OIL & \multicolumn{2}{|c|}{ SP500 } & GOLD & USD \\
\hline \multirow{2}{*}{ OIL } & -0.002 & & & & & \\
\hline & {$[0.076]$} & & & & & \\
\hline \multirow{2}{*}{ SP500 } & 0.014 & $0.215^{\star * *}$ & & & & \\
\hline & {$[0.076]$} & {$[0.073]$} & & & & \\
\hline
\end{tabular}




\section{Continued}

\begin{tabular}{cccccc}
\hline GOLD & 0.024 & 0.040 & $-0.195^{* * *}$ & & \\
& {$[0.075]$} & {$[0.076]$} & {$[0.072]$} & & \\
USD & -0.091 & -0.113 & $0.148^{* *}$ & $-0.566^{* *}$ & \\
& {$[0.075]$} & {$[0.075]$} & {$[0.075]$} & {$[0.052]$} & \\
\multirow{2}{*}{ LIBOR } & 0.045 & 0.011 & -0.021 & -0.111 & -0.018 \\
& {$[0.076]$} & {$[0.076]$} & {$[0.076]$} & {$[0.074]$} & {$[0.075]$} \\
\hline
\end{tabular}

Note: ${ }^{*}{ }^{* *}$ and ${ }^{* * *}$ denote the significance level at $10 \%, 5 \%$ and $1 \%$. Standard errors are in bracket.

Table 11. Dynamic conditional correlation MGARCH model of Stellar.

\begin{tabular}{|c|c|c|c|c|c|c|}
\hline Variables & XLM & OIL & SP500 & GOLD & USD & LIBOR \\
\hline \multirow{2}{*}{ Cons } & 0.002 & 0.0003 & $0.002^{* *}$ & 0.0001 & 0.001 & $0.003^{* * *}$ \\
\hline & {$[0.011]$} & [0.003] & {$[0.001]$} & {$[0.001]$} & {$[0.001]$} & {$[0.001]$} \\
\hline \multirow{2}{*}{ L1.ARCH } & $0.412^{\star * *}$ & $0.086^{\star}$ & $0.219^{* * *}$ & 0.062 & $0.073^{*}$ & $0.953^{* * *}$ \\
\hline & [0.139] & {$[0.052]$} & {$[0.069]$} & [0.049] & {$[0.040]$} & {$[0.180]$} \\
\hline \multirow{2}{*}{ L1.GARCH } & $0.566^{\star * *}$ & $0.788^{\star * *}$ & $0.726^{\star * \star}$ & $-0.794^{\star \star \star}$ & $0.872^{\star * *}$ & -0.006 \\
\hline & {$[0.115]$} & [0.123] & {$[0.071]$} & [0.237] & [0.087] & {$[0.008]$} \\
\hline \multirow{2}{*}{ Cons ARCH } & $0.005^{\star *}$ & 0.000 & $0.00002^{\star}$ & $0.001^{\star * *}$ & 0.000006 & $0.00006^{* * *}$ \\
\hline & {$[0.002]$} & {$[0.000]$} & {$[0.00001]$} & {$[0.0001]$} & {$[0.000007]$} & [0.000008] \\
\hline $\mathrm{N}$ & 203 & & & & & \\
\hline \multirow[t]{2}{*}{ Log likelihood } & 2769.843 & & & & & \\
\hline & \multicolumn{5}{|c|}{ Test $\mathrm{b}[$ Adjustment:lambda1 $]=\mathrm{b}[$ Adjustment:lambda2 $]=0$} & \\
\hline Chi2 & 4.53 & & & & & \\
\hline \multirow{2}{*}{ Lambda 1} & $0.046^{*}$ & & & & & \\
\hline & [0.025] & & & & & \\
\hline \multirow{2}{*}{ Lambda2 } & 0.228 & & & & & \\
\hline & [0.309] & & & & & \\
\hline Correlations & XLM & & & SP500 & GOLD & USD \\
\hline \multirow{2}{*}{ OIL } & -0.049 & & & & & \\
\hline & [0.075] & & & & & \\
\hline \multirow{2}{*}{ SP500 } & $0.136^{*}$ & & *** & & & \\
\hline & {$[0.073]$} & & & & & \\
\hline \multirow{2}{*}{ GOLD } & 0.000 & & & $-0.194^{\star * *}$ & & \\
\hline & [0.075] & & & [0.071] & & \\
\hline \multirow{2}{*}{ USD } & -0.039 & & & 0.145 & $-0.564^{* * *}$ & \\
\hline & [0.075] & & & {$[0.074]$} & {$[0.052]$} & \\
\hline \multirow{2}{*}{ LIBOR } & -0.065 & & & -0.018 & -0.115 & -0.018 \\
\hline & {$[0.076]$} & & & {$[0.075]$} & {$[0.074]$} & {$[0.074]$} \\
\hline
\end{tabular}

Note: ${ }^{*},{ }^{* *}$ and ${ }^{* *}$ denote the significance level at $10 \%, 5 \%$ and $1 \%$. Standard errors are in bracket. 
Table 12. Dynamic conditional correlation MGARCH model of Monero.

\begin{tabular}{|c|c|c|c|c|c|c|}
\hline Variables & XMR & OIL & SP500 & GOLD & USD & LIBOR \\
\hline \multirow{2}{*}{ Cons } & 0.012 & 0.00004 & $0.002^{\star \star}$ & 0.0001 & 0.001 & $0.003^{* * *}$ \\
\hline & {$[0.013]$} & {$[0.003]$} & {$[0.001]$} & {$[0.001]$} & {$[0.001]$} & {$[0.001]$} \\
\hline \multirow{2}{*}{ L1.ARCH } & $0.164^{*}$ & $0.086^{*}$ & $0.228^{\star \star \star}$ & 0.061 & $0.071^{*}$ & $0.95^{\star \star \star}$ \\
\hline & [0.09] & {$[0.051]$} & {$[0.072]$} & [0.049] & {$[0.04]$} & {$[0.178]$} \\
\hline \multirow{2}{*}{ L1.GARCH } & 0.109 & $0.784^{* * *}$ & $0.711^{\star * *}$ & $-0.792^{* * *}$ & $0.881^{* * *}$ & -0.005 \\
\hline & {$[0.205]$} & {$[0.123]$} & {$[0.072]$} & {$[0.243]$} & {$[0.086]$} & {$[0.009]$} \\
\hline \multirow{2}{*}{ Cons ARCH } & $0.024^{* * *}$ & 0.0003 & $0.00002^{*}$ & $0.001^{\star * *}$ & 0.00001 & $0.00006^{* * *}$ \\
\hline & {$[0.007]$} & {$[0.0002]$} & {$[0.00001]$} & {$[0.0001]$} & {$[0.00001]$} & {$[0.00001]$} \\
\hline $\mathrm{N}$ & 203 & & & & & \\
\hline \multirow[t]{2}{*}{ Log likelihood } & 2777.998 & & & & & \\
\hline & \multicolumn{5}{|c|}{ Test $\mathrm{b}[$ Adjustment:lambda1 $]=\mathrm{b}[$ Adjustment:lambda2 $]=0$} & \\
\hline Chi2 & $6.11^{* *}$ & & & & & \\
\hline \multirow{2}{*}{ Lambda 1} & $0.054^{\star \star}$ & & & & & \\
\hline & [0.027] & & & & & \\
\hline \multirow{2}{*}{ Lambda 2} & 0.257 & & & & & \\
\hline & {$[0.273]$} & & & & & \\
\hline Correlations & XMR & OIL & & 500 & GOLD & USD \\
\hline \multirow{2}{*}{ OIL } & -0.084 & & & & & \\
\hline & {$[0.076]$} & & & & & \\
\hline \multirow{2}{*}{ SP500 } & -0.020 & $0.203^{*}$ & & & & \\
\hline & {$[0.076]$} & {$[0.074$} & & & & \\
\hline \multirow{2}{*}{ GOLD } & 0.048 & 0.042 & & $92^{* * *}$ & & \\
\hline & [0.075] & {$[0.076$} & & 072] & & \\
\hline \multirow{2}{*}{ USD } & -0.080 & -0.10 & & $50^{* *}$ & $-0.56^{* * *}$ & \\
\hline & [0.075] & {$[0.075$} & & 075] & {$[0.053]$} & \\
\hline \multirow{2}{*}{ LIBOR } & 0.064 & 0.008 & & .024 & -0.114 & -0.014 \\
\hline & {$[0.076]$} & {$[0.076$} & & 076] & {$[0.074]$} & {$[0.075]$} \\
\hline
\end{tabular}

Note: ${ }^{*},{ }^{*}$ and ${ }^{* * *}$ denote the significance level at $10 \%, 5 \%$ and $1 \%$. Standard errors are in bracket.

Table 13. Dynamic conditional correlation MGARCH model of DASH.

\begin{tabular}{ccccccc}
\hline Variables & DAS & OIL & SP500 & GOLD & USD & LIBOR \\
\hline \multirow{2}{*}{ Cons } & 0.008 & 0.0002 & $0.002^{* *}$ & 0.0001 & 0.0007 & $0.002^{* * *}$ \\
& {$[0.011]$} & {$[0.003]$} & {$[0.001]$} & {$[0.001]$} & {$[0.001]$} & {$[0.001]$} \\
\multirow{2}{*}{ L1.ARCH } & $0.200^{* *}$ & $0.088^{*}$ & $0.239^{* * *}$ & 0.061 & $0.065^{*}$ & $0.929^{* * *}$ \\
& {$[0.091]$} & {$[0.052]$} & {$[0.074]$} & {$[0.05]$} & {$[0.038]$} & {$[0.174]$} \\
& $0.551^{* * *}$ & $0.782^{* * *}$ & $0.71^{* * *}$ & $-0.793^{* * *}$ & $0.897^{* * *}$ & -0.005 \\
L1.GARCH & {$[0.154]$} & {$[0.123]$} & {$[0.071]$} & {$[0.24]$} & {$[0.076]$} & {$[0.009]$} \\
\hline
\end{tabular}


N. P. Canh et al.

\section{Continued}

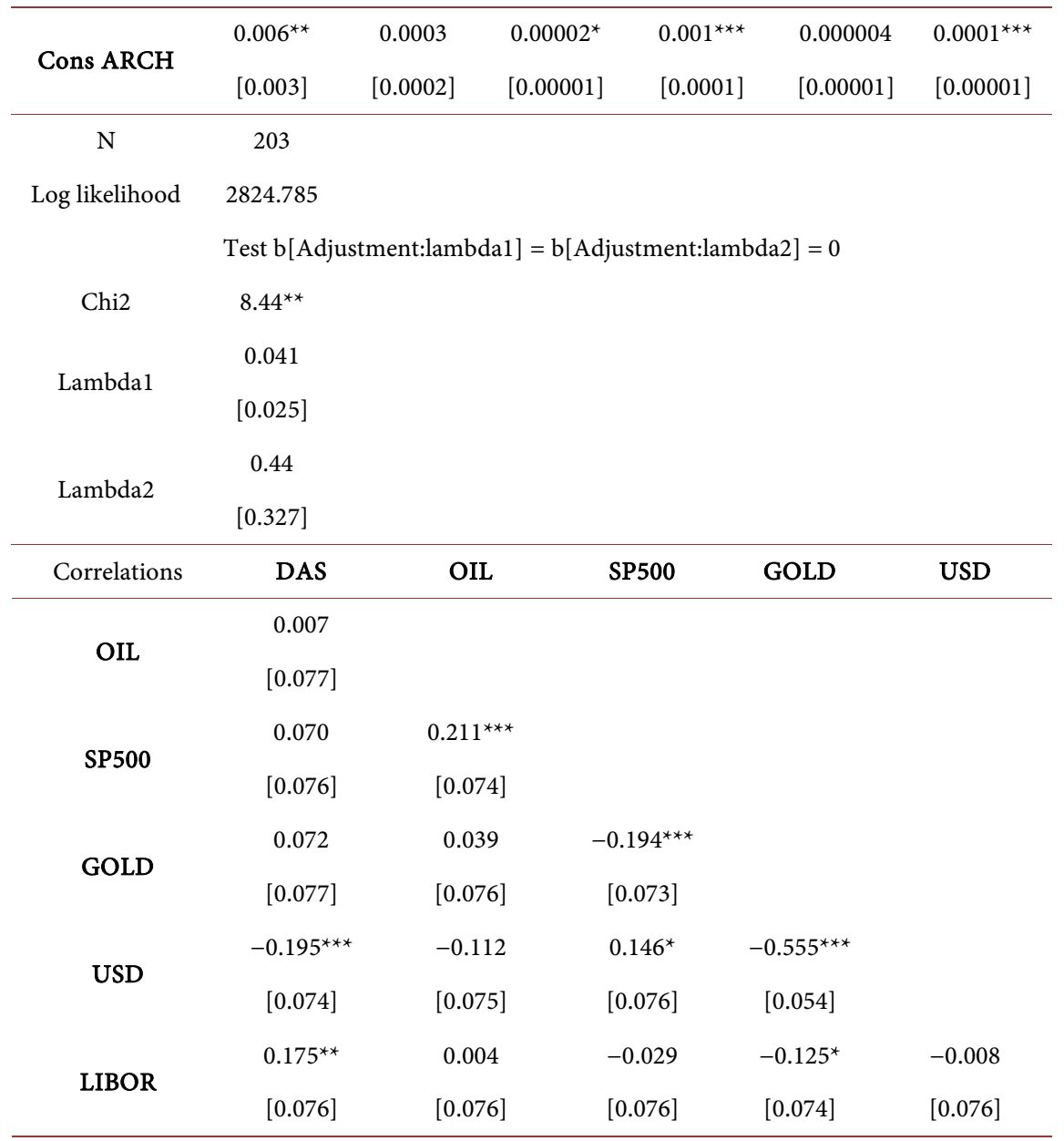

Note: ${ }^{* *}$ and ${ }^{* *}$ denote the significance level at $10 \%, 5 \%$ and $1 \%$. Standard errors are in bracket.

Table 14. Dynamic conditional correlation MGARCH model of Bytecoin.

\begin{tabular}{|c|c|c|c|c|c|c|}
\hline Variables & $\mathrm{BCN}$ & OIL & SP500 & GOLD & USD & LIBOR \\
\hline \multirow{2}{*}{ Cons } & $0.029^{* *}$ & 0.0001 & $0.002^{\star *}$ & -0.0001 & 0.001 & $0.003^{\star * *}$ \\
\hline & [0.014] & [0.003] & {$[0.001]$} & {$[0.001]$} & {$[0.001]$} & {$[0.001]$} \\
\hline \multirow{2}{*}{ L1.ARCH } & $0.308^{\star}$ & $0.083^{*}$ & $0.221^{\star * *}$ & 0.069 & $0.07^{\star}$ & $0.928^{\star * *}$ \\
\hline & [0.159] & {$[0.05]$} & {$[0.07]$} & {$[0.052]$} & {$[0.041]$} & [0.176] \\
\hline \multirow{2}{*}{ L1.GARCH } & $0.566^{\star * *}$ & $0.789^{\star * *}$ & $0.72^{\star * *}$ & $-0.769^{* * *}$ & $0.88^{* * *}$ & $-0.012^{\star * *}$ \\
\hline & {$[0.146]$} & {$[0.122]$} & {$[0.071]$} & {$[0.264]$} & {$[0.088]$} & {$[0.004]$} \\
\hline \multirow{2}{*}{ Cons ARCH } & $0.011^{\star *}$ & 0.0003 & $0.00002^{*}$ & $0.001^{\star * *}$ & 0.00001 & $0.0001^{\star * *}$ \\
\hline & {$[0.005]$} & {$[0.0002]$} & {$[0.00001]$} & {$[0.0001]$} & {$[0.00001]$} & {$[0.00001]$} \\
\hline $\mathrm{N}$ & 203 & & & & & \\
\hline \multirow[t]{2}{*}{ Log likelihood } & 2731.926 & & & & & \\
\hline & \multicolumn{6}{|c|}{ Test $\mathrm{b}[$ Adjustment:lambda1 $]=\mathrm{b}[$ Adjustment:lambda2 $]=0$} \\
\hline Chi2 & $5.83^{\star}$ & & & & & \\
\hline Lambda1 & 0.035 & & & & & \\
\hline
\end{tabular}


Continued

\begin{tabular}{cccccc}
\hline & {$[0.025]$} & & & & \\
Lambda2 & 0.395 & & & & \\
& {$[0.277]$} & & & & \\
Correlations & BCN & OIL & SP500 & GOLD & USD \\
\hline OIL & 0.065 & & & & \\
& {$[0.074]$} & & & & \\
SP500 & 0.105 & $0.215^{* * *}$ & & & \\
& {$[0.075]$} & {$[0.073]$} & & & \\
GOLD & 0.099 & 0.033 & $-0.196^{* * *}$ & & \\
& {$[0.073]$} & {$[0.076]$} & {$[0.072]$} & & \\
USD & -0.007 & -0.108 & $0.141^{*}$ & $-0.559^{* * *}$ & \\
& {$[0.074]$} & {$[0.074]$} & {$[0.075]$} & {$[0.053]$} & \\
LIBOR & -0.114 & 0.001 & -0.026 & -0.117 & -0.017 \\
& {$[0.09]$} & {$[0.075]$} & {$[0.075]$} & {$[0.074]$} & {$[0.074]$} \\
\hline
\end{tabular}

Note: ${ }^{*}{ }^{* *}$ and ${ }^{* * *}$ denote the significance levels at $10 \%, 5 \%$ and $1 \%$. Standard errors are in bracket.

\section{Conclusions}

With the assumption as financial assets, the question on the capability of cryptocurrencies in hedging to systematic risk is quite worthy to investigate. Selecting seven cryptocurrencies with largest capitalization level, the study investigates correlations between the selected cryptocurrencies and economic factors that are proxied by oil price, gold price, interest rate, USD strength, and S\&P500. Some main findings are noticeable.

First, there are strong correlations between cryptocurrencies. Moreover, there are also structural breaks and ARCH disturbance in each cryptocurrency. We suggest a systematic risk within the cryptocurrency market. Second, the Granger causality tests show that the relationship between cryptocurrencies and economic factors are undirected. Third, GARCH $(1,1)$ tests provide evidence that cryptocurrencies are insignificant correlations with economic factors with the implication that cryptocurrencies are not assumed as financial assets to hedge systematic risks. The results are robust by DCC MGARCH tests. The results are significant for financial investors on the perspective of the diversification. That is, the financial investor must be more careful in using cryptocurrencies as financial assets, especially in diversifying their portfolio since they have low capability in diversification within cryptocurrency market and also with economic risks.

\section{Acknowledgements}

This study is funded by the University of Economics Ho Chi Minh city.

\section{Conflicts of Interest}

The authors declare no conflicts of interest regarding the publication of this paper. 


\section{References}

[1] Thies, S. and Molnár, P. (2018) Bayesian Change Point Analysis of Bitcoin Returns. Finance Research Letters, 27, 223-227. https://doi.org/10.1016/j.frl.2018.03.018

[2] Gajardo, G., Kristjanpoller, W.D. and Minutolo, M. (2018) Does Bitcoin Exhibit the Same Asymmetric Multifractal Cross-Correlations with Crude Oil, Gold and DJIA as the Euro, Great British Pound and Yen? Chaos, Solitons \& Fractals, 109, 195-205. https://doi.org/10.1016/j.chaos.2018.02.029

[3] Alvarez-Ramirez, J., Rodriguez, E. and Ibarra-Valdez, C. (2018) Long-Range Correlations and Asymmetry in the Bitcoin Market. Physica A: Statistical Mechanics and Its Applications, 492, 948-955. https://doi.org/10.1016/j.physa.2017.11.025

[4] Balcilar, M., Bouri, E., Gupta, R. and Roubaud, D. (2017) Can Volume Predict Bitcoin Returns and Volatility? A Quantiles-Based Approach. Economic Modelling, 64, 74-81. https://doi.org/10.1016/j.physa.2017.11.025

[5] Brandvold, M., Molnár, P., Vagstad, K. and Andreas Valstad, O.C. (2015) Price discovery on Bitcoin Exchanges. Journal of International Financial Markets, Institutions and Money, 36, 18-35. https://doi.org/10.1016/j.intfin.2015.02.010

[6] Brauneis, A. and Mestel, R. (2018) Price Discovery of Cryptocurrencies: Bitcoin and Beyond. Economics Letters, 165, 58-61. https://doi.org/10.1016/j.econlet.2018.02.001

[7] Jiang, Y., Nie, H. and Ruan, W. (2018) Time-Varying Long-Term Memory in Bitcoin Market. Finance Research Letters, 25, 280-284. https://doi.org/10.1016/j.frl.2017.12.009

[8] Koutmos, D. (2018) Bitcoin Returns and Transaction Activity. Economics Letters, 167, 81-85. https://doi.org/10.1016/j.frl.2017.12.009

[9] Takaishi, T. (2018) Statistical Properties and Multifractality of Bitcoin. Physica A: Statistical Mechanics and Its Applications, 506, 507-519. https://doi.org/10.1016/j.physa.2018.04.046

[10] Van Vliet, B. (2018) An Alternative Model of Metcalfe's Law for Valuing Bitcoin. Economics Letters, 165, 70-72. https://doi.org/10.1016/j.econlet.2018.02.007

[11] Su, C.-W., Li, Z.-Z., Tao, R. and Si, D.-K. (2018) Testing for Multiple Bubbles in Bitcoin Markets: A Generalized Sup ADF Test. Japan and the World Economy, 46, 56-63. https://doi.org/10.1016/j.japwor.2018.03.004

[12] Baur, D. G., Dimpfl, T. and Kuck, K. (2018) Bitcoin, Gold and the US Dollar-A Replication and Extension. Finance Research Letters, 25, 103-110. https://doi.org/10.1016/j.frl.2017.10.012

[13] Cheah, E.-T. and Fry, J. (2015) Speculative Bubbles in Bitcoin Markets? An Empirical Investigation into the Fundamental Value of Bitcoin. Economics Letters, 130, 32-36. https://doi.org/10.1016/j.econlet.2015.02.029

[14] Frunza, M.-C. (2016) Chapter 1E-Cryptocurrencies: A New Monetary Vehicle. In Frunza, M.-C., Ed., Solving Modern Crime in Financial Markets, Academic Press, New York, 39-75. https://doi.org/10.1016/j.econlet.2015.02.029

[15] Fry, J. and Cheah, E.-T. (2016) Negative Bubbles and Shocks in Cryptocurrency Markets. International Review of Financial Analysis, 47, 343-352. https://doi.org/10.1016/j.irfa.2016.02.008

[16] Phillips, P.C. and Yu, J. (2011) Dating the Timeline of Financial Bubbles during the Subprime Crisis. Quantitative Economics, 2, 455-491. https://doi.org/10.3982/QE82

[17] Corbet, S., Lucey, B. and Yarovaya, L. (2017) Datestamping the Bitcoin and Ethe- 
reum Bubbles. Finance Research Letters, 26, 81-88.

https://doi.org/10.1016/j.frl.2017.12.006

[18] Gandal, N., Hamrick, J.T., Moore, T. and Oberman, T. (2018) Price Manipulation in the Bitcoin Ecosystem. Journal of Monetary Economics, 95, 86-96. https://doi.org/10.1016/j.jmoneco.2017.12.004

[19] Demir, E., Gozgor, G., Lau, C.K.M. and Vigne, S.A. (2018) Does Economic Policy Uncertainty Predict the Bitcoin Returns? An Empirical Investigation. Finance Research Letters, 26, 81-88. https://doi.org/10.1016/j.frl.2018.01.005

[20] Dyhrberg, A.H. (2016a) Bitcoin, Gold and the Dollar-A GARCH Volatility Analysis. Finance Research Letters, 16, 85-92. https://doi.org/10.1016/j.frl.2015.10.008

[21] Dyhrberg, A.H. (2016b) Hedging Capabilities of Bitcoin. Is It the Virtual Gold? Finance Research Letters, 16, 139-144. https://doi.org/10.1016/j.frl.2015.10.025

[22] Dwyer, G.P. (2015) The Economics of Bitcoin and Similar Private Digital Currencies. Journal of Financial Stability, 17, 81-91. https://doi.org/10.1016/j.jfs.2014.11.006

[23] Li, X. and Wang, C.A. (2017) The Technology and Economic Determinants of Cryptocurrency Exchange Rates: The Case of Bitcoin. Decision Support Systems, 95, 49-60. https://doi.org/10.1016/j.dss.2016.12.001

[24] Al-Yahyaee, K.H., Mensi, W. and Yoon, S.-M. (2018) Efficiency, Multifractality, and the Long-Memory Property of the Bitcoin Market: A Comparative Analysis with Stock, Currency, and Gold Markets. Finance Research Letters, 27, 228-234. https://doi.org/10.1016/j.frl.2018.03.017

[25] Ciaian, P., Rajcaniova, M. and Kancs, D.A. (2018) Virtual Relationships: Short- and Long-Run Evidence from BitCoin and Altcoin Markets. Journal of International Financial Markets, Institutions and Money, 52, 173-195. https://doi.org/10.1016/j.intfin.2017.11.001

[26] Chen, Y. (2018) Blockchain Tokens and the Potential Democratization of Entrepreneurship and Innovation. Business Horizons. https://doi.org/10.1016/j.bushor.2018.03.006

[27] Acatrinei, M., Gorun, A. and Marcu, N. (2013) A Dcc-Garch Model to Estimate. Romanian Journal of Economic Forecasting, 1, 136-148.

[28] Engle, R. (2002) Dynamic Conditional Correlation: A Simple Class of Multivariate Generalized Autoregressive Conditional Heteroskedasticity Models. Journal of Business \& Economic Statistics, 20, 339-350. https://doi.org/10.1198/073500102288618487

[29] Peters, T. (2008) Forecasting the Covariance Matrix with the DCC GARCH Model.

[30] Billio, M., Caporin, M. and Gobbo, M. (2006) Flexible Dynamic Conditional Correlation Multivariate Garch Models for asset allocation. Applied Financial Economics Letters, 2, 123-130. https://doi.org/10.1080/17446540500428843

[31] Lee, M.-C., Chiou, J.-S. and Lin, C.-M. (2006) A Study of Value-at-Risk on Portfolio in Stock Return Using DCC Multivariate GARCH. Applied Financial Economics Letters, 2, 183-188. https://doi.org/10.1080/17446540500447645 